\title{
A Study of Behavior of the Sequence of Norm of Primitives of Functions in Orlicz Spaces Depending on Their Spectrum
}

\author{
Ha Huy BANG and Vu Nhat HUY \\ Vietnamese Academy of Science and Technology and Vietnam National University \\ (Communicated by K. Takemura)
}

Abstract. In this paper we characterize behavior of the sequence of norm of primitives of functions in Orlicz spaces by its spectrum (the support of its Fourier transform).

\section{Introduction}

The following result was proved in [13]:

THEOREM A. Let $1 \leq p \leq \infty, f \in L_{p}\left(\mathbb{R}^{n}\right), f \not \equiv 0$ and $\operatorname{supp} \hat{f}$ is bounded, where $\hat{f}$ is the Fourier transform of $f$. Then

$$
\lim _{|\alpha| \rightarrow \infty}\left(\frac{\left\|D^{\alpha} f\right\|_{p}}{\sup _{\xi \in \operatorname{supp} \hat{f}}\left|\xi^{\alpha}\right|}\right)^{1 /|\alpha|}=1 .
$$

The novelty and importance of Theorem A is that it is almost impossible to calculate directly the sequence $\left\{\left\|D^{\alpha} f\right\|_{p}^{1 /|\alpha|}\right\}$ but we can approximately calculate it by calculating the sequence $\left\{\sup _{\xi \in \operatorname{supp} f}\left|\xi^{\alpha}\right|^{1 /|\alpha|}\right\}$ and this is possible if we know all "far from the origin" points of "supp $\hat{f}$." This result was proved for $n=1$ in [12] and was studied and developed by many authors (see [1]-[17], [22], [24]-[27], [36]-[38]). It is natural to ask what will happen when we replace derivatives by integrals. To this question, V.K. Tuan answered in [39] for $p=2, n=1$, we answered for $1 \leq p \leq \infty, n=1$ in [20] and for $1 \leq p \leq \infty, n \geq 1$ in [21], and we answer now for the $n$-dimensional case and Orlicz spaces.

Received January 16, 2014; revised June 12, 2014 


\section{Definitions}

Let $\Phi:[0, \infty) \rightarrow[0, \infty]$ be an arbitrary Young function, i.e., $\Phi(0)=0, \Phi(t) \geq$ $0, \Phi(t) \not \equiv 0$ and $\Phi$ is convex. Denote by:

$$
\bar{\Phi}(t)=\sup _{s \geq 0}\{t s-\Phi(s)\} \quad(0 \leq t<\infty)
$$

the Young function conjugate to $\Phi$ and $L_{\Phi}\left(\mathbb{R}^{n}\right)$-the space of measurable function $u$ such that

$$
|\langle u, v\rangle|=\left|\int_{\mathbb{R}^{n}} u(x) v(x) d x\right|<\infty
$$

for all $v$ with $\rho(v, \bar{\Phi})<\infty$, where

$$
\rho(v, \bar{\Phi})=\int_{\mathbb{R}^{n}} \bar{\Phi}(|v(x)|) d x .
$$

Then $L_{\Phi}\left(\mathbb{R}^{n}\right)$ is a Banach space with respect to the Orlicz norm

$$
\|u\|_{\Phi}=\|u\|_{L_{\Phi}\left(\mathbb{R}^{n}\right)}=\sup _{\rho(v, \bar{\Phi}) \leq 1}\left|\int_{\mathbb{R}^{n}} u(x) v(x) d x\right|,
$$

which is equivalent to the Luxemburg norm

$$
\|f\|_{(\Phi)}=\inf \left\{\lambda>0: \int_{\mathbb{R}^{n}} \Phi(|f(x)| / \lambda) d x \leq 1\right\} .
$$

Note that Lebesgue spaces and their extension - Orlicz spaces play an important role in Analysis and have many applications (see [23], [29]-[35]). Recall that $\|\cdot\|_{(\Phi)}=\|\cdot\|_{p}$ where $\Phi(t)=t^{p}$ with $1 \leq p<\infty$, and $\|\cdot\|_{(\Phi)}=\|\cdot\|_{\infty}$ where $\Phi(t)=0$ for $0 \leq t \leq 1$ and $\Phi(t)=\infty$ for $t>1$.

We have known the following results:

Lemma 1. Let $u \in L_{\Phi}\left(\mathbb{R}^{n}\right)$ and $v \in L_{\bar{\Phi}}\left(\mathbb{R}^{n}\right)$. Then

$$
\int_{\mathbb{R}^{n}}|u(x) v(x)| d x \leq\|u\|_{\Phi}\|v\|_{\bar{\Phi}} .
$$

LemMA 2. Let $u \in L_{\Phi}\left(\mathbb{R}^{n}\right)$ and $v \in L_{1}\left(\mathbb{R}^{n}\right)$. Then

$$
\|u * v\|_{\Phi} \leq\|v\|_{\Phi}\|v\|_{1} .
$$

\section{Mail results}

We shall first give a notation of the primitive of a tempered generalized function: Denote by $S\left(\mathbb{R}^{n}\right)$ the Schwartz space of rapidly decreasing functions and $S^{\prime}\left(\mathbb{R}^{n}\right)$ the set of all 
continuous linear functionals on $S\left(\mathbb{R}^{n}\right)$. Any element $h \in S^{\prime}\left(\mathbb{R}^{n}\right)$ is called a tempered generalized function and we write $h(\varphi)=\langle h, \varphi\rangle$ for all $\varphi \in S\left(\mathbb{R}^{n}\right)$. Let $f \in S^{\prime}\left(\mathbb{R}^{n}\right)$ and $e_{j}=(0, \ldots, 0,1,0, \ldots, 0) \in \mathbb{Z}_{+}^{n}$ be an unit vector such that its $j^{\text {th }}$ coordinate equals 1 , $j=1,2, \ldots, n$. The tempered generalized function $I^{e_{j}} f$ is termed an $e_{j}^{t h}$ primitive of $f$ if $D^{e_{j}}\left(I^{e_{j}} f\right)=f$, that is,

$$
\left\langle I^{e_{j}} f, D^{e_{j}} \varphi\right\rangle=-\langle f, \varphi\rangle \quad \forall \varphi \in S\left(\mathbb{R}^{n}\right),
$$

where $D^{e_{j}} f$ is the $e_{j}^{t h}$ derivative of $f$. Now we show that for each $f \in S^{\prime}\left(\mathbb{R}^{n}\right)$ the set

$$
\mathcal{P}^{e_{j}}(f)=\left\{h \in S^{\prime}\left(\mathbb{R}^{n}\right): \quad D^{e_{j}} h=f\right\}
$$

is not empty. Indeed, for $\varphi \in S\left(\mathbb{R}^{n}\right)$ we define the following functions

$$
\begin{aligned}
& \psi(x)=\varphi(x)-\theta\left(x_{j}\right) \int_{-\infty}^{\infty} \varphi\left(x_{1}, \ldots, x_{j-1}, t, x_{j+1}, \ldots, x_{n}\right) d t, \\
& \Psi(x)=\int_{-\infty}^{x_{j}} \psi\left(x_{1}, \ldots, x_{j-1}, t, x_{j+1}, \ldots, x_{n}\right) d t
\end{aligned}
$$

where

$$
\theta \in C_{0}^{\infty}(\mathbb{R}), \operatorname{supp} \theta \subset[-1,1], \int_{\mathbb{R}} \theta(t) d t=1
$$

Then, it is easy to see that $\psi, \Psi \in S\left(\mathbb{R}^{n}\right)$. So, for each $f \in S^{\prime}\left(\mathbb{R}^{n}\right)$, we can define the tempered generalized function $J^{e_{j}} f$ via the formula

$$
\left\langle J^{e_{j}} f, \varphi\right\rangle=-\langle f, \Psi\rangle .
$$

Then $J^{e_{j}} f \in S^{\prime}\left(\mathbb{R}^{n}\right)$ and

$$
\left\langle J^{e_{j}} f, D^{e_{j}} \varphi\right\rangle=-\left\langle f, \int_{-\infty}^{x_{j}} D^{e_{j}} \varphi\left(x_{1}, \ldots, x_{j-1}, t, x_{j+1}, \ldots, x_{n}\right) d t\right\rangle=-\langle f, \varphi\rangle .
$$

Therefore, $J^{e_{j}} f \in \mathcal{P}^{e_{j}}(f)$. Furthermore, let $I^{e_{j}} f \in \mathcal{P}^{e_{j}}(f)$, we have

$$
\begin{aligned}
\left\langle I^{e_{j}} f, \varphi\right\rangle & =\left\langle I^{e_{j}} f(x), D^{e_{j}} \Psi(x)+\theta\left(x_{j}\right) \int_{\mathbb{R}} \varphi\left(x_{1}, \ldots, x_{j-1}, t, x_{j+1}, \ldots, x_{n}\right) d t\right\rangle \\
& =\left\langle I^{e_{j}} f(x), D^{e_{j}} \Psi(x)\right\rangle+\left\langle I^{e_{j}} f(x), \theta\left(x_{j}\right) \int_{\mathbb{R}} \varphi\left(x_{1}, \ldots, x_{j-1}, t, x_{j+1}, \ldots, x_{n}\right) d t\right\rangle \\
& =-\langle f, \Psi\rangle+\left\langle I^{e_{j}} f(x), \theta\left(x_{j}\right) \int_{\mathbb{R}} \varphi\left(x_{1}, \ldots, x_{j-1}, t, x_{j+1}, \ldots, x_{n}\right) d t\right\rangle .
\end{aligned}
$$

Hence,

$$
\left\langle I^{e_{j}} f, \varphi\right\rangle=-\left\langle J^{e_{j}} f, \varphi\right\rangle+\left\langle g_{j}, \varphi\right\rangle,
$$


where $g_{j} \in S^{\prime}\left(\mathbb{R}^{n}\right)$ is defined by

$$
\left\langle g_{j}, \varphi\right\rangle=\left\langle f_{j}\left(x_{1}, \ldots, x_{j-1}, x_{j+1}, \ldots, x_{n}\right), \int_{\mathbb{R}} \varphi\left(x_{1}, \ldots, x_{j-1}, t, x_{j+1}, \ldots, x_{n}\right) d t\right\rangle
$$

with $f_{j} \in S^{\prime}\left(\mathbb{R}^{n-1}\right)$ is defined by $\left\langle f_{j}, h\right\rangle=\left\langle I^{e_{j}} f(x), \theta\left(x_{j}\right) h\left(x_{1}, \ldots, x_{j-1}, x_{j+1}, \ldots, x_{n}\right)\right\rangle$ for each $h \in S\left(\mathbb{R}^{n-1}\right)$. Then $g_{j}$ is called the $j^{\text {th }}$ constant tempered generalized function. Note that $g_{j}$ becomes a constant if $n=1$.

Conversely, given an arbitrary the $j^{\text {th }}$ constant tempered generalized function $g_{j}$, the functional $I^{e_{j}} f$ defined on $S\left(\mathbb{R}^{n}\right)$ by (1) determines the $e_{j}^{t h}$ primitive of $f$. So, we have proved the following result: Let $j \in\{1, \ldots, n\}$. Every tempered generalized function $f \in S^{\prime}\left(\mathbb{R}^{n}\right)$ has in $S^{\prime}\left(\mathbb{R}^{n}\right)$ the $e_{j}^{\text {th }}$ primitive, which is denoted by $I^{e_{j}} f$, and every $e_{j}^{\text {th }}$ primitive of $f$ is expressed by formula (1), where $g_{j}$ is an arbitrary the $j^{\text {th }}$ constant tempered generalized function.

Note that the notation of primitive of a generalized function in $D^{\prime}(a, b), a, b \in \mathbb{R}$ can be found in [40], here we define it for tempered generalized functions in $S^{\prime}\left(\mathbb{R}^{n}\right)$. We denote $I^{0} f=f$. In the sequel, for $j=1,2, \ldots, n$ then $I^{e_{j}} f$ denotes the $e_{j}^{t h}$ primitive of $f \in$ $S^{\prime}\left(\mathbb{R}^{n}\right)$, i.e., $I^{e_{j}} f \in \mathcal{P}^{e_{j}}(f)$, and for any $\alpha \in \mathbb{Z}_{+}^{n},|\alpha| \geq 1$ we define the primitive of higher order by the following way: $I^{\alpha} f=I^{e_{j_{\alpha}}}\left(I^{\alpha-e_{j_{\alpha}}} f\right)$, where $j_{\alpha}:=\max \left\{j: \alpha_{j} \geq 1\right\}$, i.e., $I^{\alpha} f \in \mathcal{P}^{e_{j_{\alpha}}}\left(I^{\alpha-e_{j_{\alpha}}} f\right)$. So, $D^{\alpha}\left(I^{\alpha} f\right)=f$ for all $\alpha \in \mathbb{Z}_{+}^{n}$. Let $1 \leq p \leq \infty$ and $f \in L_{\Phi}\left(\mathbb{R}^{n}\right)$. If for any $\alpha \in \mathbb{Z}_{+}^{n}$ there exists the $e_{j_{\alpha}}$ primitive of $I^{\alpha-e_{j \alpha}} f$, which belongs to $L_{\Phi}\left(\mathbb{R}^{n}\right)$ and is denoted by $I^{\alpha} f$, we write $\left(I^{\alpha} f\right)_{\alpha \in \mathbb{Z}_{+}^{n}} \subset L_{\Phi}\left(\mathbb{R}^{n}\right)$.

Before giving our main results, we introduce a notation of the extended convolution of functions with different numbers of variables:

Definition 3. Let $n \geq k, f$ be a locally integrable function in $\mathbb{R}^{n}, g$ be a locally integrable functions in $\mathbb{R}^{k}$. If the integral

$$
\int_{\mathbb{R}^{k}} f\left(x_{1}-y_{1}, \ldots, x_{k}-y_{k}, x_{k+1}, \ldots, x_{n}\right) . g\left(y_{1}, \ldots, y_{k}\right) d y_{1} \ldots d y_{k}
$$

exists for almost all $x=\left(x_{1}, \ldots, x_{n}\right)$, then it is called the extended convolution of $f$ and $g$, and is symbolized as $(f \circledast g)(x)$.

The above definition becomes the known one if $n=k$. For the extended convolution we have the following result:

Proposition 4. Let $n, k \in \mathbb{N}, n \geq k, f \in L_{\Phi}\left(\mathbb{R}^{n}\right)$ and $g \in L_{1}\left(\mathbb{R}^{k}\right)$. Then $f \circledast g \in$ $L_{\Phi}\left(\mathbb{R}^{n}\right)$ and

$$
\|f \circledast g\|_{L_{\Phi}\left(\mathbb{R}^{n}\right)} \leq\|f\|_{L_{\Phi}\left(\mathbb{R}^{n}\right)}\|g\|_{L_{1}\left(\mathbb{R}^{k}\right)} .
$$


Proof. Put $\mathbf{v}=\left(x_{k+1}, \ldots, x_{n}\right)$ and denote $f\left(x_{1}, \ldots, x_{k}, \mathbf{v}\right):=f\left(x_{1}, \ldots, x_{n}\right)$. Then by the definition of Orlicz norm we obtain

$$
\|f \circledast g\|_{L_{\Phi}\left(\mathbb{R}^{n}\right)}=\sup \left\{|\langle f \circledast g, \varphi\rangle|: \quad\|\varphi\|_{(\bar{\Phi})} \leq 1\right\} .
$$

Note that

$$
\begin{aligned}
\langle f \circledast g, \varphi\rangle & =\int_{\mathbb{R}^{n}}\left(\int_{\mathbb{R}^{k}} f\left(x_{1}-y_{1}, \ldots, x_{k}-y_{k}, \mathbf{v}\right) g\left(y_{1}, \ldots, y_{k}\right) d y_{1} \ldots d y_{k}\right) \varphi\left(x_{1}, \ldots, x_{n}\right) d x \\
& =\int_{\mathbb{R}^{k}}\left(\int_{\mathbb{R}^{n}} f\left(x_{1}-y_{1}, \ldots, x_{k}-y_{k}, \mathbf{v}\right) \varphi\left(x_{1}, \ldots, x_{n}\right) d x\right) g\left(y_{1}, \ldots, y_{k}\right) d y_{1} \ldots d y_{k} \\
& =\int_{\mathbb{R}^{k}} F\left(y_{1}, \ldots, y_{k}\right) g\left(y_{1}, \ldots, y_{k}\right) d y_{1} \ldots d y_{k},
\end{aligned}
$$

where

$$
F\left(y_{1}, \ldots, y_{k}\right)=\int_{\mathbb{R}^{n}} f\left(x_{1}-y_{1}, \ldots, x_{k}-y_{k}, \mathbf{v}\right) \varphi\left(x_{1}, \ldots, x_{n}\right) d x
$$

Then

$$
F\left(y_{1}, \ldots, y_{k}\right)=\int_{\mathbb{R}^{n}} f_{\left(y_{1}, \ldots, y_{k}\right)}\left(x_{1}, \ldots, x_{n}\right) \varphi\left(x_{1}, \ldots, x_{n}\right) d x
$$

with $f_{\left(y_{1}, \ldots, y_{k}\right)}\left(x_{1}, \ldots, x_{n}\right)=f\left(x_{1}-y_{1}, \ldots, x_{k}-y_{k}, \mathbf{v}\right)$, and then it follows from Lemma 1 that

$$
\left|F\left(y_{1}, \ldots, y_{k}\right)\right| \leq\left\|f_{\left(y_{1}, \ldots, y_{k}\right)}\right\|_{L_{\Phi}\left(\mathbb{R}^{n}\right)}\|\varphi\|_{(\bar{\Phi})}=\|f\|_{L_{\Phi}\left(\mathbb{R}^{n}\right)}\|\varphi\|_{(\bar{\Phi})} .
$$

Therefore,

$$
|\langle f \circledast g, \varphi\rangle| \leq\|f\|_{L_{\Phi}\left(\mathbb{R}^{n}\right)}\|g\|_{L_{1}\left(\mathbb{R}^{k}\right)}\|\varphi\|_{(\bar{\Phi})} .
$$

So,

$$
\|f \circledast g\|_{L_{\Phi}\left(\mathbb{R}^{n}\right)} \leq\|f\|_{L_{\Phi}\left(\mathbb{R}^{n}\right)}\|g\|_{L_{1}\left(\mathbb{R}^{k}\right)} .
$$

The proof is complete.

Let $f \in L_{1}\left(\mathbb{R}^{n}\right)$ and $\hat{f}=F f$ or $F_{n} f$ be its Fourier transform

$$
\hat{f}(\xi)=(2 \pi)^{-n / 2} \int_{\mathbb{R}^{n}} e^{-i x \xi} f(x) d x .
$$

The Fourier transform of a tempered generalized function $f$ is defined via the formula

$$
\langle F f, \varphi\rangle=\langle f, F \varphi\rangle, \quad \varphi \in S\left(\mathbb{R}^{n}\right) .
$$


Let $K$ be an arbitrary compact set in $\mathbb{R}^{n}$ and $\varepsilon>0$. Denote by $B(x, \varepsilon):=\left\{\xi \in \mathbb{R}^{n}\right.$ : $\left.\|x-\xi\|=\left(\sum_{k=1}^{n}\left(x_{k}-\xi_{k}\right)^{2}\right)^{1 / 2}<\varepsilon\right\}-$ the ball of radius $\varepsilon>0$ with center at the point $x$, $B[x, \varepsilon]:=\left\{\xi \in \mathbb{R}^{n}: \quad\|x-\xi\| \leq \varepsilon\right\}, K_{\varepsilon}:=\left\{\xi \in \mathbb{R}^{n}: \quad \exists x \in K:\|x-\xi\| \leq \varepsilon\right\},|x|=$ $\sum_{j=1}^{n}\left|x_{j}\right|$. We put $\left(\mathbb{R}^{n}, \varepsilon\right):=\left\{\xi \in \mathbb{R}^{n}: \min \left\{\left|\xi_{1}\right|,\left|\xi_{2}\right|, \ldots,\left|\xi_{n}\right|\right\}>\varepsilon\right\}$.

The extended convolution has the following property which is essential to prove our results:

Proposition 5. Let $n, k \in \mathbb{N}, n \geq k, f \in S\left(\mathbb{R}^{n}\right)$ and $g \in L_{1}\left(\mathbb{R}^{k}\right)$. Then

(3) $\left(F_{n}\left(f\left(\xi_{1}, \ldots, \xi_{n}\right) g\left(\xi_{1}, \ldots, \xi_{k}\right)\right)\right)\left(x_{1}, \ldots, x_{n}\right)=(2 \pi)^{-k / 2}\left(F_{n} f \circledast F_{k} g\right)\left(x_{1}, \ldots, x_{n}\right)$,

$$
\left(F_{n}^{-1}\left(f\left(\xi_{1}, \ldots, \xi_{n}\right) g\left(\xi_{1}, \ldots, \xi_{k}\right)\right)\right)\left(x_{1}, \ldots, x_{n}\right)=(2 \pi)^{-k / 2}\left(F_{n}^{-1} f \circledast F_{k}^{-1} g\right)\left(x_{1}, \ldots, x_{n}\right)
$$

ProOF. Put

$$
u=\left(x_{1}, x_{2}, \ldots, x_{k}\right), v=\left(x_{k+1}, \ldots, x_{n}\right), s=\left(\xi_{1}, \xi_{2}, \ldots, \xi_{k}\right), t=\left(\xi_{k+1}, \ldots, \xi_{n}\right) .
$$

Then

$$
x=(u, v), \xi=(s, t), d \xi=d s d t, x \xi=u s+v t
$$

Therefore,

$$
\begin{aligned}
\left(F_{n}\left(f\left(\xi_{1}, \ldots, \xi_{n}\right) g\left(\xi_{1}, \ldots, \xi_{k}\right)\right)\right)(x) & =(2 \pi)^{-n / 2} \int_{\mathbb{R}^{n}} e^{-i(u s+v t)} f(s, t) g(s) d s d t \\
& =(2 \pi)^{-n / 2} \int_{\mathbb{R}^{k}}\left[e^{-i u s} g(s) \int_{\mathbb{R}^{n-k}} e^{-i v t} f(s, t) d t\right] d s
\end{aligned}
$$

and then

$$
\left(F_{n}\left(f\left(\xi_{1}, \ldots, \xi_{n}\right) g\left(\xi_{1}, \ldots, \xi_{k}\right)\right)\right)(x)=(2 \pi)^{-k / 2} \int_{\mathbb{R}^{k}} e^{-i u s} g(s) G_{v}(s) d s
$$

where

$$
G_{v}(s)=(2 \pi)^{-(n-k) / 2} \int_{\mathbb{R}^{n-k}} e^{-i v t} f(s, t) d t
$$

Hence,

$$
\left(F_{n}\left(f\left(\xi_{1}, \ldots, \xi_{n}\right) g\left(\xi_{1}, \ldots, \xi_{k}\right)\right)\right)(x)=(2 \pi)^{-k / 2}\left(F_{k} G_{v}\right) *\left(F_{k}(g)\right)(u) .
$$


We see that

$$
\begin{aligned}
\left(F_{k} G_{v}\right)(u) & =(2 \pi)^{-k / 2} \int_{\mathbb{R}^{k}} e^{-i u s} G_{v}(s) d s \\
& =(2 \pi)^{-n / 2} \int_{\mathbb{R}^{k}}\left(e^{-i u s} \int_{\mathbb{R}^{n-k}} e^{-i v t} f(s, t) d t\right) d s=(2 \pi)^{-n / 2} \int_{\mathbb{R}^{n}} e^{-i x \xi} f(\xi) d \xi .
\end{aligned}
$$

That means

$$
\left(F_{k} G_{v}\right)(u)=\left(F_{n} f\right)(x) .
$$

Combining (4)-(5), we arrive at (3). Similarly, we also have the result for the inverse Fourier transform. The proof is complete.

It is easy to see the following property of the extended convolution:

Proposition 6. Let $n, k \in \mathbb{N}, n \geq k, f \in L_{\Phi}\left(\mathbb{R}^{n}\right), h \in S\left(\mathbb{R}^{n}\right)$ and $g \in L_{1}\left(\mathbb{R}^{k}\right)$. Then

$$
\langle f \circledast g, h\rangle=\langle f, h \circledast g(-x)\rangle
$$

Let $f \in S^{\prime}\left(\mathbb{R}^{n}\right)$. Then $\operatorname{supp} \hat{f}$ is called the spectrum of $f$. We say that $f$ has $(O)$ property if its spectrum is contained in $\left(\mathbb{R}^{n}, \Delta\right)$ for some $\Delta>0$. Using the extended convolution we have the following result on the existence of primitives in $L_{\Phi}\left(\mathbb{R}^{n}\right)$ :

THEOREM 7. Let $f \in L_{\Phi}\left(\mathbb{R}^{n}\right)$ and $f$ has $(O)$-property. Then for any $j=1, \ldots, n$ there exists exactly one $e_{j}^{\text {th }}$ primitive of $f$, which is denoted by $I^{e_{j}} f$, such that $I^{e_{j}} f \in L_{\Phi}\left(\mathbb{R}^{n}\right)$ and also has $(O)$-property. Moreover, $\operatorname{supp} \widehat{I^{e_{j} f}}=\operatorname{supp} \hat{f}$.

PROOF. It is sufficient to prove for the case $j=1$. By the assumption, supp $\hat{f} \subset$ $\left(\mathbb{R}^{n}, \Delta\right)$ for some $\Delta>0$. We define a function $\eta \in C^{\infty}(\mathbb{R})$ by

$$
\eta(x)=\frac{\phi(x)}{(-i x)^{3}}
$$

where the even function $\phi \in C^{\infty}(\mathbb{R})$ is given and satisfies the following conditions

$$
\begin{array}{ll}
\phi(x)=1 \quad \forall x \in(-\infty,-\Delta / 2) \cup(\Delta / 2,+\infty), \\
\phi(x)=0 \quad \forall x \in(-\Delta / 4, \Delta / 4) .
\end{array}
$$

Then it follows from (7) that the function $\eta$ is well defined and it is not difficult to see that $\sup _{x \in \mathbb{R}}\left(1+x^{2}\right)|\hat{\eta}(x)|<\infty$. So, $\hat{\eta} \in L_{1}(\mathbb{R})$ and then the following function $\Psi$ is well defined via the formula

$$
\Psi\left(x_{1}, \ldots, x_{n}\right)=\frac{1}{\sqrt{2 \pi}} \int_{\mathbb{R}} f\left(x_{1}-\xi, x_{2}, \ldots, x_{n}\right) \hat{\eta}(\xi) d \xi
$$


Then it follows from Proposition $4, f \in L_{\Phi}\left(\mathbb{R}^{n}\right)$ and $\hat{\eta} \in L_{1}(\mathbb{R})$ that $\Psi \in L_{\Phi}\left(\mathbb{R}^{n}\right)$. For each $\varphi \in S\left(\mathbb{R}^{n}\right)$, we put $\psi=D^{3 e_{1}} \varphi$. Then

$$
\begin{aligned}
\langle\Psi, \psi\rangle & =\int_{\mathbb{R}^{n}} \Psi\left(x_{1}, \ldots, x_{n}\right) \psi\left(x_{1}, \ldots, x_{n}\right) d x \\
& =\frac{1}{\sqrt{2 \pi}} \int_{\mathbb{R}^{n}}\left(\int_{\mathbb{R}} f\left(x_{1}-\xi, x_{2}, \ldots, x_{n}\right) \hat{\eta}(\xi) d \xi\right) \psi\left(x_{1}, \ldots, x_{n}\right) d x \\
& =\frac{1}{\sqrt{2 \pi}} \int_{\mathbb{R}^{n}}\left(\int_{\mathbb{R}} f\left(\xi, x_{2}, \ldots, x_{n}\right) \hat{\eta}\left(x_{1}-\xi\right) d \xi\right) \psi\left(x_{1}, \ldots, x_{n}\right) d x \\
& =\frac{1}{\sqrt{2 \pi}} \int_{\mathbb{R}^{n}}\left(\int_{\mathbb{R}} \psi\left(x_{1}, x_{2}, \ldots, x_{n}\right) \hat{\eta}\left(x_{1}-\xi\right) d x_{1}\right) f\left(\xi, x_{2}, \ldots, x_{n}\right) d \xi d x_{2} \ldots, d x_{n} \\
& =-\langle f, g\rangle,
\end{aligned}
$$

where

$$
g\left(\xi, x_{2}, \ldots, x_{n}\right)=\frac{1}{\sqrt{2 \pi}} \int_{\mathbb{R}} \psi\left(x_{1}, x_{2}, \ldots, x_{n}\right) \hat{\eta}\left(\xi-x_{1}\right) d x_{1}
$$

Hence,

$$
g\left(x_{1}, \ldots, x_{n}\right)=\frac{1}{\sqrt{2 \pi}} \int_{\mathbb{R}} \psi\left(x_{1}-\xi, x_{2}, \ldots, x_{n}\right) \hat{\eta}(\xi) d \xi .
$$

So, applying Proposition 5, we get

$$
\left(F^{-1} g\right)\left(x_{1}, \ldots, x_{n}\right)=\left(F^{-1} \psi\right)\left(x_{1}, \ldots, x_{n}\right) \eta\left(x_{1}\right)=\left(F^{-1} \varphi\right)\left(x_{1}, \ldots, x_{n}\right) \phi\left(x_{1}\right) .
$$

From (6) and supp $\hat{f} \subset\left(\mathbb{R}^{n}, \Delta\right)$, we have $\hat{f}=\hat{f} \phi\left(x_{1}\right)$. Therefore, since (8), we obtain

$$
\begin{aligned}
\langle f, g\rangle & =\left\langle\hat{f}, F^{-1} g\right\rangle=\left\langle\hat{f},\left(F^{-1} \varphi\right)\left(x_{1}, \ldots, x_{n}\right) \phi\left(x_{1}\right)\right\rangle \\
& =\left\langle\hat{f} \phi\left(x_{1}\right),\left(F^{-1} \varphi\right)\left(x_{1}, \ldots, x_{n}\right)\right\rangle=\left\langle\hat{f},\left(F^{-1} \varphi\right)\left(x_{1}, \ldots, x_{n}\right)\right\rangle=\langle f, \varphi\rangle .
\end{aligned}
$$

This implies

$$
\left\langle\Psi, D^{3 e_{1}} \varphi\right\rangle=-\langle f, \varphi\rangle .
$$

So, $D^{3 e_{1}} \Psi=f$ in the distribution sense. Hence, we have $D^{3 e_{1}} \Psi \in L_{\Phi}\left(\mathbb{R}^{n}\right)$ and $\Psi \in$ $L_{\Phi}\left(\mathbb{R}^{n}\right)$. Therefore, $D^{2 e_{1}} \Psi \in L_{\Phi}\left(\mathbb{R}^{n}\right)$, and it is symbolized as $I^{e_{1}} f$.

Next, we prove that $\operatorname{supp} \widehat{I^{e_{1}} f}=\operatorname{supp} \hat{f}$. Indeed, since $D^{e_{1}} I^{e_{1}} f=f$, we have

$$
\hat{f}=i x_{1} \widehat{I^{e_{1}} f} \text {. }
$$


Therefore,

$$
\operatorname{supp} \hat{f} \subset \operatorname{supp} \widehat{I^{e_{1}} f} \subset \operatorname{supp} \hat{f} \cup H_{1},
$$

where $H_{1}:=\left\{\xi \in \mathbb{R}^{n}: \quad \xi_{1}=0\right\}$. So, to prove supp $\widehat{I^{\alpha} f}=\operatorname{supp} \hat{f}$, it is enough to show $H_{1} \cap \operatorname{supp} \widehat{I^{e_{1}} f}=\{\emptyset\}$. Assume now the contrary that $\exists \xi \in H_{1} \cap \widehat{I^{e_{1}} f}$. Then $b:=$ $\max \left\{\left|\xi_{1}\right|, \ldots,\left|\xi_{n}\right|\right\}>0$. We choose a number $0<a<\Delta$ and a function $h \in C_{0}^{\infty}((-\Delta, \Delta) \times$ $\left.(-b-a-1, b+a+1)^{n-1}\right)$ such that $h(x)=1$ in $(-a, a) \times(-b-a, b+a)^{n-1}$. Since (9) and supp $\hat{f} \subset\left(\mathbb{R}^{n}, \Delta\right)$, we have

$$
\operatorname{supph} \widehat{I^{e_{1}} f} \subset H_{1} .
$$

Hence, there is a number $N_{0} \in \mathbb{N}$ such that

$$
F^{-1} h * I^{e_{1}} f(x)=\sum_{j=0}^{N_{0}} g_{j}^{N_{0}}\left(x_{2}, \ldots, x_{n}\right)\left(-i x_{1}\right)^{j} .
$$

From Lemma 1 and $I^{e_{1}} f \in L_{\Phi}\left(\mathbb{R}^{n}\right), F^{-1} h \in L_{\bar{\Phi}}\left(\mathbb{R}^{n}\right)$, we get $F^{-1} h * I^{e_{1}} f \in L_{\infty}\left(\mathbb{R}^{n}\right)$. Therefore,

$$
F^{-1} h * I^{e_{1}} f(x)=g_{0}^{N_{0}}\left(x_{2}, \ldots, x_{n}\right) .
$$

By the same manner, we also have

$$
F^{-1} h * \Psi(x)=g_{0}^{N_{1}}\left(x_{2}, \ldots, x_{n}\right) .
$$

Note that

$$
\begin{aligned}
F^{-1} h * I^{e_{1}} f(x) & =F^{-1} h * D^{2 e_{1}} \Psi(x) \\
& =D^{2 e_{1}}\left(F^{-1} h * \Psi(x)\right)=D^{2 e_{1}}\left(g_{0}^{N_{1}}\left(x_{2}, \ldots, x_{n}\right)\right) \equiv 0 .
\end{aligned}
$$

So, $h \widehat{I^{e_{1}} f}=0$. On the other hand, since $\xi \in \operatorname{supp} \widehat{I^{e_{1}} f}$, there is a function $\varphi \in C_{0}^{\infty}(B(\xi, a))$ such that

$$
\left\langle\widehat{I^{e_{1}} f}, \varphi\right\rangle \neq 0 .
$$

Then, since $h(x)=1$ in $(-a, a) \times(-b-a, b+a)^{n-1}$, we get

$$
0 \neq\left\langle\widehat{I^{e_{1}} f}, \varphi\right\rangle=\left\langle\widehat{I^{e_{1}} f}, h \varphi\right\rangle=\left\langle h \widehat{I^{e_{1}} f}, \varphi\right\rangle=0 .
$$

This is impossible. Hence, $\operatorname{supp} \widehat{e^{e_{1}} f}=\operatorname{supp} \hat{f}$.

Finally, we prove the uniqueness of the $e_{1}^{t h}$ primitive of $f$ which belongs to $L_{\Phi}\left(\mathbb{R}^{n}\right)$ and has $(O)$-property. Indeed, suppose that $I^{e_{1}} f$ and $J^{e_{1}} f$ are certain $e_{1}^{t h}$ primitives of $f$ such that $I^{e_{1}} f, J^{e_{1}} f \in L_{\Phi}\left(\mathbb{R}^{n}\right)$ and both of them have $(O)$-property. Since $I^{e_{1}} f, J^{e_{1}} f \in L_{\Phi}\left(\mathbb{R}^{n}\right)$ and $D^{e_{1}}\left(J^{e_{1}} f-I^{e_{1}} f\right)=0$, we get $J^{e_{1}} f-I^{e_{1}} f=g\left(x_{2}, \ldots, x_{n}\right)$. Hence,

$$
\left\langle F\left(J^{e_{1}} f-I^{e_{1}} f\right), \eta\left(x_{1}\right) \varphi\left(x_{2}, \ldots, x_{n}\right)\right\rangle=\eta(0)\left\langle g\left(x_{2}, \ldots, x_{n}\right), F_{n-1}\left(\varphi\left(x_{2}, \ldots, x_{n}\right)\right)\right\rangle,
$$


where $\varphi \in S\left(\mathbb{R}^{n-1}\right)$ and $\eta \in C_{0}^{\infty}(\mathbb{R})$. So,

$$
\left\{(0, \sigma) \in \mathbb{R}^{n}: \quad \sigma \in \operatorname{supp} F_{n-1}\left(g\left(x_{2}, \ldots, x_{n}\right)\right)\right\} \subset \operatorname{supp} F\left(J^{e_{1}} f-I^{e_{1}} f\right) .
$$

On the other hand, it follows from $\operatorname{supp} F\left(J^{e_{1}} f-I^{e_{1}} f\right) \subset \operatorname{supp} F\left(J^{e_{1}} f\right) \cup \operatorname{supp} F\left(I^{e_{1}} f\right)$ that $J^{e_{1}} f-I^{e_{1}} f$ has $(O)$-property. Therefore, $\operatorname{supp} F_{n-1}\left(g\left(x_{2}, \ldots, x_{n}\right)\right)=\emptyset$, i.e., $J^{e_{1}} f-I^{e_{1}} f=$ 0 a.e. The proof is complete.

REMARK 8. Let an Young function $\Phi:[0, \infty) \rightarrow[0, \infty)$ and $f \in L_{\Phi}\left(\mathbb{R}^{n}\right)$. Then for any $j=1, \ldots, n$ there is at most one in $L_{\Phi}\left(\mathbb{R}^{n}\right)$ the $e_{j}^{t h}$ primitive of $f$, which is denoted by $I^{e_{j}} f$, and moreover, there exists exactly one in $L_{\Phi}\left(\mathbb{R}^{n}\right)$ the $e_{j}^{t h}$ primitive of $f$ if $f$ has $(O)$-property.

\section{REMARK 9.}

1. If $f \in L_{\Phi}\left(\mathbb{R}^{n}\right)$ doesn't have $(O)$-property then it is possible that there doesn't exist in $L_{\Phi}\left(\mathbb{R}^{n}\right)$ any the $e_{j}^{t h}$ primitive of $f, j=1,2, \ldots, n$. It is illustrated in the following examples:

+) $L_{\Phi}\left(\mathbb{R}^{n}\right)=L_{\infty}\left(\mathbb{R}^{n}\right)$ and $f \equiv 1($ then $\operatorname{supp} \hat{f}=\{0\})$;

+) $L_{\Phi}\left(\mathbb{R}^{n}\right)=L_{\infty}\left(\mathbb{R}^{n}\right)$ and $f(x)=\prod_{j=1}^{n} \sin ^{2} x_{j}$ (then $\operatorname{supp} \hat{f}=\left\{x \in \mathbb{R}^{n}: x_{j} \in\right.$ $\{0,-2,2\}, j=1,2, . ., n\})$;

+) $\Phi(t)=t^{p}$ with $1 \leq p<\infty$ and $f(x)=\prod_{j=1}^{n} \sin ^{2} x_{j} / x_{j}^{2}\left(\right.$ then supp $\left.\hat{f}=[-2,2]^{n}\right)$.

2. Let $L_{\Phi}\left(\mathbb{R}^{n}\right)=L_{\infty}\left(\mathbb{R}^{n}\right)$ and $f, I^{\alpha} f \in L_{\infty}\left(\mathbb{R}^{n}\right)$. Put $g(x)=f(x)+c$, where $0 \neq c$ is a constant. Then $g \in L_{\infty}\left(\mathbb{R}^{n}\right)$ but there doesn't exist the $\alpha^{t h}$ primitive $I^{\alpha} g \in L_{\infty}\left(\mathbb{R}^{n}\right)$.

3. Let $L_{\Phi}\left(\mathbb{R}^{n}\right)=L_{\infty}\left(\mathbb{R}^{n}\right)$ and $f(x)=\prod_{j=1}^{n} \cos x_{j}$. Then supp $\hat{f}=\left\{x \in \mathbb{R}^{n}: x_{j} \in\right.$ $\{-1,1\}, j=1,2, \ldots, n\}$ and there exists in $L_{\infty}\left(\mathbb{R}^{n}\right)$ any $I^{\alpha} f$ - the $\alpha^{t h}$ primitive of $f$.

THEOREM 10. Let $f \in L_{\Phi}\left(\mathbb{R}^{n}\right)$. Then there is at most one sequence of primitives $\left(I^{\alpha} f\right)_{\alpha \in \mathbb{Z}_{+}^{n}} \subset L_{\Phi}\left(\mathbb{R}^{n}\right)$.

PROOF. We have to show that if there exists another sequence of primitives of $f$, which

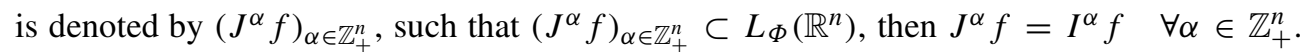
Indeed, it is sufficient to prove this fact for the case $\alpha=e_{1}$. Since $J^{e_{1}} f, I^{e_{1}} f, f \in L_{\Phi}\left(\mathbb{R}^{n}\right)$ and $D^{e_{1}}\left(J^{e_{1}} f-I^{e_{1}} f\right)=0$, we get

$$
J^{e_{1}} f-I^{e_{1}} f=g\left(x_{2}, \ldots, x_{n}\right)
$$

and then

$$
D^{e_{1}}\left(J^{2 e_{1}} f-I^{2 e_{1}} f\right)=g\left(x_{2}, \ldots, x_{n}\right) .
$$


Hence,

$$
J^{2 e_{1}} f-I^{2 e_{1}} f=x_{1} g\left(x_{2}, \ldots, x_{n}\right)+h\left(x_{2}, \ldots, x_{n}\right),
$$

where $h\left(x_{2}, \ldots, x_{n}\right)$ is another generalized function independent on $x_{1}$. Therefore, taking account of $J^{2 e_{1}} f-I^{2 e_{1}} f \in L_{\Phi}\left(\mathbb{R}^{n}\right)$, we get $g\left(x_{2}, \ldots, x_{n}\right)=0$ a.e. So, $J^{e_{1}} f=I^{e_{1}} f$. The proof is complete.

Using Theorems 7 and 10, we have the following:

THEOREM 11. Let $f \in L_{\Phi}\left(\mathbb{R}^{n}\right)$ and $f$ has $(O)$-property. Then there exists exactly one sequence of primitives $\left(I^{\alpha} f\right)_{\alpha \in \mathbb{Z}_{+}^{n}} \subset L_{\Phi}\left(\mathbb{R}^{n}\right)$. Moreover, $\operatorname{supp} \widehat{I^{\alpha} f}=\operatorname{supp} \hat{f} \quad \forall \alpha \in \mathbb{Z}_{+}^{n}$.

Now, we characterize behavior of the sequence of $L_{\Phi}\left(\mathbb{R}^{n}\right)$-norm of primitives of a function by its spectrum:

THEOREM 12. Let $f \not \equiv 0, f$ has $(O)$-property and $\left(I^{\alpha} f\right)_{\alpha \in \mathbb{Z}_{+}^{n}} \subset L_{\Phi}\left(\mathbb{R}^{n}\right)$. Then

$$
\lim _{|\alpha| \rightarrow \infty}\left(\left(\inf _{\xi \in \operatorname{supp} \hat{f}}\left|\xi^{\alpha}\right|\right)\left\|I^{\alpha} f\right\|_{\Phi}\right)^{1 /|\alpha|}=1
$$

To prove Theorem 12, we need the following result:

Proposition 13. Let $h \in C^{\infty}\left(\mathbb{R}^{n}\right)$ satisfy supph $\subset\left(\mathbb{R}^{n}, \Delta\right)$ for some $\Delta>0$ and $\max \left\{\left\|D^{\beta} h\right\|_{\infty}: \beta \in \mathbb{Z}_{+}^{n}, \beta \leq(3,3, \ldots, 3)\right\}<\infty$. We put

$$
A=\left\{\alpha \in \mathbb{Z}_{+}^{n}: \alpha_{j} \geq 3, j=1, \ldots, n\right\} .
$$

Then for any $\alpha \in A$ the function $H_{\alpha}(x)=F\left(h(\xi) / \xi^{\alpha}\right)$ is well defined and

$$
\text { (A) } \varlimsup_{|\alpha| \rightarrow \infty}\left(\left(\inf _{\xi \in \operatorname{supp} h}\left|\xi^{\alpha}\right|\right)\left\|H_{\alpha}\right\|_{1}\right)^{1 /|\alpha|} \leq 1,
$$

where (A) stands before the upper limit means that we take the limit only for $\alpha \in A$.

PROOF. For $\xi=\left(\xi_{1}, \xi_{2}, \ldots, \xi_{n}\right)$, we adopt the convention that $\xi^{2}=\prod_{j=1}^{n} \xi_{j}^{2}$ and $\xi-2=\left(\xi_{1}-2, \ldots, \xi_{n}-2\right)$. Since supph $\subset\left(\mathbb{R}^{n}, \Delta\right)$ for some $\Delta>0$ and $\alpha \in A$, it follows that $h(\xi) / \xi^{\alpha} \in L_{1}\left(\mathbb{R}^{n}\right)$ and then $H_{\alpha}$ is well defined. So,

$$
H_{\alpha}(x)=(2 \pi)^{-n / 2} \int_{\mathbb{R}^{n}} e^{-i x \xi} \frac{h(\xi)}{\xi^{\alpha}} d \xi=(2 \pi)^{-n / 2} \int_{\xi \in \operatorname{supp} h} e^{-i x \xi} \frac{h(\xi)}{\xi^{\alpha}} d \xi .
$$

Hence, for $\beta \in \mathbb{Z}_{+}^{n}, \beta \leq(2,2, \ldots, 2)$ we have the following estimate

$$
\sup _{x \in \mathbb{R}^{n}}\left|x^{\beta} H_{\alpha}(x)\right| \leq(2 \pi)^{-n / 2} \sup _{x \in \mathbb{R}^{n}}\left|\int_{\mathbb{R}^{n}} e^{-i x \xi} D^{\beta}\left(\frac{h(\xi)}{\xi^{\alpha}}\right) d \xi\right|
$$




$$
\begin{aligned}
& =(2 \pi)^{-n / 2} \sup _{x \in \mathbb{R}^{n}}\left|\int_{\xi \in \operatorname{supp} h} e^{-i x \xi} D^{\beta}\left(\frac{h(\xi)}{\xi^{\alpha}}\right) d \xi\right| \\
& \leq(2 \pi)^{-n / 2} \int_{\xi \in \operatorname{supp} h}\left|D^{\beta}\left(\frac{h(\xi)}{\xi^{\alpha}}\right)\right| d \xi .
\end{aligned}
$$

Then it follows from the Leibniz rule that

$$
\text { (12) } \begin{aligned}
\sup _{x \in \mathbb{R}^{n}} & \left|x^{\beta} H_{\alpha}(x)\right| \leq(2 \pi)^{-n / 2} \int_{\xi \in \operatorname{supp} h}\left|\sum_{\gamma \leq \beta} \frac{\beta !}{\gamma !(\beta-\gamma) !} D^{\gamma} h(\xi) D^{\beta-\gamma}\left(\frac{1}{\xi^{\alpha}}\right)\right| d \xi \\
& \leq(2 \pi)^{-n / 2} \sum_{\gamma \leq \beta}\left(\frac{\beta !}{\gamma !(\beta-\gamma) !} \sup _{x \in \operatorname{supp} h}\left|x^{2} D^{\beta-\gamma}\left(\frac{1}{x^{\alpha}}\right)\right| \int_{\xi \in \operatorname{supp} h}\left|\frac{1}{\xi^{2}} D^{\gamma} h(\xi)\right| d \xi\right) \\
& \leq(2 \pi)^{-n / 2} \max _{\theta \leq(2,2, \ldots, 2)} \sup _{x \in \operatorname{supp} h}\left|x^{2} D^{\theta}\left(\frac{1}{x^{\alpha}}\right)\right| \sum_{\gamma \leq \beta}\left(\frac{\beta !}{\gamma !(\beta-\gamma) !} \int_{\xi \in\left(\mathbb{R}^{n}, \Delta\right)} C\left|\frac{1}{\xi^{2}}\right| d \xi\right) \\
& \leq(2 \pi)^{-n / 2} \max _{\theta \leq(2,2, \ldots, 2)} \sup _{x \in \operatorname{supp} h}\left|x^{2} D^{\theta}\left(\frac{1}{x^{\alpha}}\right)\right| \frac{C 2^{3 n}}{\Delta^{n}},
\end{aligned}
$$

where $C=\max \left\{\left\|D^{\beta} h\right\|_{\infty}: \beta \in \mathbb{Z}_{+}^{n}, \beta \leq(3,3, \ldots, 3)\right\}$.

Since supph $\subset\left(\mathbb{R}^{n}, \Delta\right)$, there exists a constant $C_{1}$ not depending on $\alpha$ such that

$$
\sup _{x \in \operatorname{supph}}\left|x^{2} D^{\theta}\left(\frac{1}{x^{\alpha}}\right)\right| \leq C_{1}|\alpha|^{2 n} \sup _{x \in \operatorname{supp} h}\left|\frac{1}{x^{\alpha-2}}\right|, \quad \forall \theta \in \mathbb{Z}_{+}^{n}, \theta \leq(2,2, \ldots, 2) .
$$

From (12)-(13), we have

$$
\sup _{x \in \mathbb{R}^{n}}\left|x^{\beta} H_{\alpha}(x)\right| \leq(2 \pi)^{-n / 2} C_{1}|\alpha|^{2 n} \sup _{x \in \operatorname{supp} h}\left|\frac{1}{x^{\alpha-2}}\right| \frac{C 2^{3 n}}{\Delta^{n}} .
$$

Let $0 \leq k \leq n$ and $\left(i_{1}, i_{2}, \ldots, i_{n}\right)$ be a permutation of $(1,2, \ldots, n)$. We define $A\left(i_{1}, \ldots, i_{n}, k\right)=\left\{x \in \mathbb{R}^{n}:\left|x_{i_{1}}\right| \geq 1, \ldots,\left|x_{i_{k}}\right| \geq 1,\left|x_{i_{k+1}}\right| \leq 1, \ldots,\left|x_{i_{n}}\right| \leq 1\right\}$. Then it follows from (14) and

$$
\begin{aligned}
\int_{\mathbb{R}^{n}}\left|H_{\alpha}(x)\right| d x & \leq \sum_{\left(i_{1}, \ldots, i_{n}, k\right)} \int\left|H_{A\left(i_{1}, \ldots, i_{n}, k\right)}(x)\right| d x \\
& \leq \sum_{\left(i_{1}, \ldots, i_{n}, k\right)} \sup _{x \in \mathbb{R}^{n}}\left|x_{i_{1}}^{2} \ldots x_{i_{k}}^{2} H_{\alpha}(x)\right| \int_{A\left(i_{1}, \ldots, i_{n}, k\right)}\left|\frac{1}{x_{i_{1}}^{2} \ldots x_{i_{k}}^{2}}\right| d x \\
& \leq 2^{n} \sum_{\left(i_{1}, \ldots, i_{n}, k\right)} \sup _{x \in \mathbb{R}^{n}}\left|x_{i_{1}}^{2} \ldots x_{i_{k}}^{2} H_{\alpha}(x)\right| \\
& \leq(2(n+1))^{n} \sup _{\left(i_{1}, \ldots, i_{n}, k\right)} \sup _{x \in \mathbb{R}^{n}}\left|x_{i_{1}}^{2} \ldots x_{i_{k}}^{2} H_{\alpha}(x)\right|
\end{aligned}
$$


that

$$
\text { (A) } \varlimsup_{|\alpha| \rightarrow \infty}\left(\left(\inf _{\xi \in \operatorname{supp} h}\left|\xi^{\alpha-2}\right|\right)\left\|H_{\alpha}\right\|_{1}\right)^{1 /|\alpha|} \leq 1
$$

Now we prove

$$
\lim _{r \rightarrow \sigma} \inf _{\xi \in \operatorname{supp} h}\left|\xi^{r}\right|=\inf _{\xi \in \operatorname{supph}}\left|\xi^{\sigma}\right|, \quad r, \sigma \geq(0,0, \ldots, 0),
$$

where $\left|\xi^{r}\right|:=\prod_{j=1}^{n}\left|\xi_{j}\right|^{r_{j}}$. Indeed, given $\sigma \geq(0,0, \ldots, 0)$ and $\lambda>1$, there exists a positive number $\varepsilon$ such that $\lambda r \geq \sigma$ for all $r \geq(0,0, \ldots, 0)$ satisfying $|r-\sigma|<\varepsilon$. Therefore,

$$
\left|\xi^{r}\right|=\left|\xi^{r-(\sigma / \lambda)}\right|\left(\left|\xi^{\sigma}\right|\right)^{1 / \lambda} \geq \Delta^{|r-(\sigma / \lambda)|}\left(\left|\xi^{\sigma}\right|\right)^{1 / \lambda} \quad \forall \xi \in \operatorname{supp} h
$$

Thus we have obtained

$$
\inf _{\xi \in \operatorname{supp} h}\left|\xi^{r}\right| \geq \Delta^{|r-(\sigma / \lambda)|}\left(\left|\xi^{\sigma}\right|\right)^{1 / \lambda}
$$

Letting $r \rightarrow \sigma$, we get

$$
\varliminf_{r \rightarrow \sigma} \inf _{\xi \in \text { supph }}\left|\xi^{r}\right| \geq \Delta^{|\sigma(1-(1 / \lambda))|}\left(\left|\xi^{\sigma}\right|\right)^{1 / \lambda} .
$$

Letting $\lambda \rightarrow 1^{+}$, we have

$$
\varliminf_{r \rightarrow \sigma} \inf _{\xi \in \text { supph }}\left|\xi^{r}\right| \geq \inf _{\xi \in \operatorname{supp} h}\left|\xi^{\sigma}\right| .
$$

On the other hand, for all $\varepsilon>0$ there exists $\xi_{\varepsilon} \in \operatorname{supph}$ such that

$$
\inf _{\xi \in \text { supph }}\left|\xi^{\sigma}\right| \leq\left|\xi_{\varepsilon}^{\sigma}\right| \leq \inf _{\xi \in \text { supph }}\left|\xi^{\sigma}\right|+\varepsilon .
$$

Taking account of

$$
\inf _{\xi \in \operatorname{supph}}\left|\xi^{r}\right| \leq\left|\xi_{\varepsilon}^{r}\right|
$$

and letting $r \rightarrow \sigma$, we have

$$
\varlimsup_{r \rightarrow \sigma} \inf _{\xi \in \text { supph }}\left|\xi^{r}\right| \leq\left|\xi_{\varepsilon}^{\sigma}\right| \leq \inf _{\xi \in \text { supph }}\left|\xi^{\sigma}\right|+\varepsilon
$$

and then

$$
\varlimsup_{r \rightarrow \sigma} \inf _{\xi \in \operatorname{supp} h}\left|\xi^{r}\right| \leq \inf _{\xi \in \text { supph }}\left|\xi^{\sigma}\right|
$$

by letting $\varepsilon \rightarrow 0$.

Combining (17) and (18), we get (16).

Now, assume the contrary that (11) is false. Then there exist an unbounded subset $I \subset A$, a number $\lambda>1$ and a vector $\beta \geq 0,|\beta|=1$ such that

$$
\text { (I) } \lim _{|\alpha| \rightarrow \infty} \frac{\alpha}{|\alpha|}=\beta
$$




$$
\text { (I) } \lim _{|\alpha| \rightarrow \infty}\left(\left(\inf _{\xi \in \operatorname{supp} h}\left|\xi^{\alpha}\right|\right)\left\|H_{\alpha}\right\|_{1}\right)^{1 /|\alpha|} \geq \lambda .
$$

Since (I) $\lim _{|\alpha| \rightarrow \infty} \frac{\alpha}{|\alpha|}=\beta$ and supph $\subset\left(\mathbb{R}^{n}, \Delta\right)$, it follows from (16) that

$$
\text { (I) } \lim _{|\alpha| \rightarrow \infty}\left(\inf _{\xi \in \operatorname{supp} h}\left|\xi^{\alpha}\right|\right)^{1 /|\alpha|}=\inf _{\xi \in \operatorname{supp} h}\left|\xi^{\beta}\right|
$$

Taking account of $(I) \lim _{|\alpha| \rightarrow \infty} \frac{\alpha-2}{|\alpha|}=\beta$, supph $\subset\left(\mathbb{R}^{n}, \Delta\right)$ and (16), we get

$$
\text { (I) } \lim _{|\alpha| \rightarrow \infty}\left(\inf _{\xi \in \operatorname{supp} h}\left|\xi^{\alpha-2}\right|\right)^{1 /|\alpha|}=\inf _{\xi \in \operatorname{supph}}\left|\xi^{\beta}\right| .
$$

Combining (19)-(20), we have

$$
\text { (I) } \lim _{|\alpha| \rightarrow \infty}\left(\inf _{\xi \in \operatorname{supph}}\left|\xi^{\alpha}\right|\right)^{1 /|\alpha|}=(I) \lim _{|\alpha| \rightarrow \infty}\left(\inf _{\xi \in \operatorname{supph}}\left|\xi^{\alpha-2}\right|\right)^{1 /|\alpha|} .
$$

Therefore, since (15), we obtain

$$
\text { (I) } \varlimsup_{|\alpha| \rightarrow \infty}\left(\left(\inf _{\xi \in \operatorname{supp} h}\left|\xi^{\alpha}\right|\right)\left\|H_{\alpha}\right\|_{1}\right)^{1 /|\alpha|} \leq 1 .
$$

This is a contradiction. So, (11) has been proved. The proof is complete.

Further, we need the following result $[16,17]$ :

THEOREM B. Let $f \in L_{\Phi}\left(\mathbb{R}^{n}\right), f \not \equiv 0$ and $\operatorname{supp} \hat{f}$ be compact. Then

$$
\lim _{|\alpha| \rightarrow \infty}\left(\frac{\left\|D^{\alpha} f\right\|_{\Phi}}{\sup _{\xi \in \operatorname{supp} \hat{f}}\left|\xi^{\alpha}\right|}\right)^{1 /|\alpha|}=1 .
$$

Proof of Theorem 12. Put $K:=\operatorname{supp} \hat{f}$. Since $f$ has (O)-property, supp $\hat{f} \subset$ $\left(\mathbb{R}^{n}, \Delta\right)$ for some $\Delta>0$. From Theorem 11 we have

$$
\operatorname{supp} \widehat{I^{\alpha} f}=K \quad \forall \alpha \in \mathbb{Z}_{+}^{n} .
$$

Now we show

$$
D^{\beta}\left(I^{\alpha+\beta} f\right)=I^{\alpha} f \quad \forall \alpha, \beta \in \mathbb{Z}_{+}^{n} .
$$

Indeed, we choose a function $g \in C^{\infty}\left(\mathbb{R}^{n}\right)$ satisfying $g(x)=1$ for all $x \in K_{\Delta / 4}$ and $g(x)=0$ for all $x \notin K_{\Delta / 2}$. For any $\varphi \in C_{0}^{\infty}\left(\mathbb{R}^{n}\right)$ it follows from (21) and supp $g \subset\left(\mathbb{R}^{n}, \Delta / 2\right)$ that

$$
\begin{gathered}
\left\langle\hat{f},\left(F^{-1} \varphi\right)(x) g(x) /(i x)^{\alpha}\right\rangle=\left\langle(i x)^{\alpha} \widehat{I^{\alpha} f},\left(F^{-1} \varphi\right)(x) g(x) /(i x)^{\alpha}\right\rangle \\
=\left\langle\widehat{I^{\alpha} f},\left(F^{-1} \varphi\right)(x) g(x)\right\rangle=\left\langle g(x) \widehat{I^{\alpha} f},\left(F^{-1} \varphi\right)(x)\right\rangle=\left\langle I^{\alpha} f, \varphi\right\rangle .
\end{gathered}
$$


Similarly,

$$
\left\langle\hat{f},\left(F^{-1}\left(D^{\beta} \varphi\right)\right)(x) g(x) /(i x)^{\alpha+\beta}\right\rangle=\left\langle I^{\alpha+\beta} f, D^{\beta} \varphi\right\rangle
$$

and then

$$
\left\langle I^{\alpha+\beta} f, D^{\beta} \varphi\right\rangle=(-1)^{|\beta|}\left\langle\hat{f},\left(F^{-1} \varphi\right)(x) g(x) /(i x)^{\alpha}\right\rangle .
$$

Using (23)-(24), we have $\left\langle I^{\alpha+\beta} f, D^{\beta} \varphi\right\rangle=(-1)^{|\beta|}\left\langle I^{\alpha} f, \varphi\right\rangle$ and then (22) have been proved.

Next we prove that

$$
\varlimsup_{|\alpha| \rightarrow \infty}\left(\left(\inf _{\xi \in \operatorname{supp} \hat{f}}\left|\xi^{\alpha}\right|\right) \cdot\left\|I^{\alpha} f\right\|_{\Phi}\right)^{1 /|\alpha|} \leq 1 .
$$

To obtain (25), we divide our proof into three steps.

Step 1. We show

$$
\left(A_{0}\right) \varlimsup_{|\alpha| \rightarrow \infty}\left(\left(\inf _{\xi \in \operatorname{supp} \hat{f}}\left|\xi^{\alpha}\right|\right) \cdot\left\|I^{\alpha} f\right\|_{\Phi}\right)^{1 /|\alpha|} \leq 1,
$$

where $A_{0}:=\left\{\alpha \in \mathbb{Z}_{+}^{n}: \quad \alpha_{j} \geq 3\right.$ for all $\left.j=1, \ldots, n\right\}$. Indeed, given an arbitrary number $\varepsilon \in(0, \Delta / 2)$. We choose a functions $\rho(x) \in C_{0}^{\infty}(B(0,1))$ such that $\int_{\mathbb{R}^{n}} \rho(x) d x=1$ and put

$$
u(x)=1_{K_{3 \varepsilon / 4}}(x), h(x)=\left(u * \rho_{\varepsilon / 4}\right)(x),
$$

where $\rho_{\varepsilon / 4}(x)=\left(\frac{4}{\varepsilon}\right)^{n} \rho\left(\frac{4}{\varepsilon} x\right)$. Then the function $h \in C^{\infty}\left(\mathbb{R}^{n}\right)$ satisfies the following conditions

$$
\begin{array}{ll}
h\left(x_{1}, \ldots, x_{n}\right)=1 & \forall\left(x_{1}, \ldots, x_{n}\right) \in K_{\varepsilon / 2}, \\
h\left(x_{1}, \ldots, x_{n}\right)=0 & \forall\left(x_{1}, \ldots, x_{n}\right) \notin K_{\varepsilon}, \\
\max \left\{\left\|D^{\beta} h\right\|_{\infty}:\right. & \beta \leq(3,3, \ldots, 3)\}<\infty .
\end{array}
$$

From (26) and the fact that $\hat{f}=(i \xi)^{\alpha} \widehat{I^{\alpha} f}$, we get $h(\xi) \hat{f}=(i \xi)^{\alpha} \widehat{I^{\alpha} f}$. So,

$$
\hat{f} h(\xi) /(i \xi)^{\alpha}=\widehat{I^{\alpha} f}
$$

and then for $\alpha \in A_{0}$

$$
\left|I^{\alpha} f\right|=(2 \pi)^{-n / 2}\left|f * F^{-1}\left(h(\xi) / \xi^{\alpha}\right)\right| .
$$

Therefore, it follows from Lemma 2 that for $\alpha \in A_{0}$ :

$$
\left\|I^{\alpha} f\right\|_{\Phi} \leq(2 \pi)^{-n / 2}\|f\|_{\Phi}\left\|F^{-1}\left(h(\xi) / \xi^{\alpha}\right)\right\|_{1}=(2 \pi)^{-n / 2}\|f\|_{\Phi}\left\|F\left(h(\xi) / \xi^{\alpha}\right)\right\|_{1} .
$$

Using (27)-(28) and Proposition 13, we have

$$
\left(A_{0}\right) \varlimsup_{|\alpha| \rightarrow \infty}\left(\left(\inf _{\xi \in \operatorname{supp} h}\left|\xi^{\alpha}\right|\right)\left\|F\left(h(\xi) / \xi^{\alpha}\right)\right\|_{1}\right)^{1 /|\alpha|} \leq 1 .
$$


Hence,

$$
\left(A_{0}\right) \varlimsup_{|\alpha| \rightarrow \infty}\left(\left(\inf _{\xi \in K_{\varepsilon}}\left|\xi^{\alpha}\right|\right)\left\|F\left(h(\xi) / \xi^{\alpha}\right)\right\|_{1}\right)^{1 /|\alpha|} \leq 1 .
$$

Therefore, by (29), we obtain

$$
\left(A_{0}\right) \varlimsup_{|\alpha| \rightarrow \infty}\left(\left(\inf _{\xi \in K_{\varepsilon}}\left|\xi^{\alpha}\right|\right)\left\|I^{\alpha} f\right\|_{\Phi}\right)^{1 /|\alpha|} \leq 1 .
$$

Since $K \subset\left(\mathbb{R}^{n}, \Delta\right)$, it is easy to check that

$$
\left(\inf _{\xi \in K_{\varepsilon}}\left|\xi^{\alpha}\right|\right)^{1 /|\alpha|} \geq\left(\inf _{\xi \in K}\left|\xi^{\alpha}\right|\right)^{1 /|\alpha|} \frac{\Delta-\varepsilon}{\Delta} .
$$

Combining (30) and (31), we get

$$
\left(A_{0}\right) \varlimsup_{|\alpha| \rightarrow \infty}\left(\left(\inf _{\xi \in \operatorname{supp} \hat{f}}\left|\xi^{\alpha}\right|\right)\left\|I^{\alpha} f\right\|_{\Phi}\right)^{1 /|\alpha|} \leq \frac{\Delta}{\Delta-\varepsilon} .
$$

Letting $\varepsilon \rightarrow 0$, we have

$$
\left(A_{0}\right) \varlimsup_{|\alpha| \rightarrow \infty}\left(\left(\inf _{\xi \in \operatorname{supp} \hat{f}}\left|\xi^{\alpha}\right|\right)\left\|I^{\alpha} f\right\|_{\Phi}\right)^{1 /|\alpha|} \leq 1 .
$$

Step 2. We prove that

$$
\left(A_{k}\right) \varlimsup_{|\alpha| \rightarrow \infty}\left(\left(\inf _{\xi \in \operatorname{supp} \hat{f}}\left|\xi^{\alpha}\right|\right)\left\|I^{\alpha} f\right\|_{\Phi}\right)^{1 /|\alpha|} \leq 1,
$$

where $A_{k}:=\left\{\alpha \in \mathbb{Z}_{+}^{n}: \quad \alpha_{1}, \ldots, \alpha_{k} \geq 3, \alpha_{k+1}=\cdots=\alpha_{n}=0\right\}$. Put

$$
\alpha^{\prime}:=\left(\alpha_{1}, \ldots, \alpha_{k}\right), K^{\prime}:=\left\{\xi \in \mathbb{R}^{k}: \quad(\xi, u) \in K \text { for some } u \in \mathbb{R}^{n-k}\right\},
$$

and we define a function $h_{1}(x) \in C^{\infty}\left(\mathbb{R}^{k}\right)$ satisfying the following conditions

$$
\begin{array}{ll}
h_{1}\left(x_{1}, \ldots, x_{k}\right)=1 & \forall\left(x_{1}, \ldots, x_{k}\right) \in K_{\varepsilon / 2}^{\prime} \\
h_{1}\left(x_{1}, \ldots, x_{k}\right)=0 & \forall\left(x_{1}, \ldots, x_{k}\right) \notin K_{\varepsilon}^{\prime} \\
\max \left\{\left\|D^{\beta} h_{1}\right\|_{\infty}:\right. & \left.\beta \in \mathbb{Z}_{+}^{k}, \beta \leq(3,3, \ldots, 3)\right\}<\infty .
\end{array}
$$

Using (32) and the fact that $\hat{f}=(i \xi)^{\alpha} \widehat{I^{\alpha} f}$, we obtain $h(\xi) \hat{f}=(i \xi)^{\alpha} \widehat{I^{\alpha} f}$. So,

$$
\hat{f} h(\xi) /(i \xi)^{\alpha}=\widehat{I^{\alpha} f} .
$$

Therefore, it follows from Proposition 4 and Proposition 6 we can deduce for $\alpha \in A_{k}, \varphi \in$ $S\left(\mathbb{R}^{n}\right)$ that

$$
\left|\left\langle f \circledast F_{k}^{-1}\left(h_{1}\left(x_{1}, \ldots, x_{k}\right) /\left(x_{1}^{\alpha_{1}} \ldots, x_{k}^{\alpha_{k}}\right)\right), \varphi\right\rangle\right|=\left|\left\langle\widehat{I^{\alpha} f}, \varphi\right\rangle\right| .
$$


So,

$$
\left|I^{\alpha} f\right|=(2 \pi)^{-k / 2}\left|f \circledast F_{k}^{-1}\left(h_{1}\left(x_{1}, \ldots, x_{k}\right) /\left(x_{1}^{\alpha_{1}} \ldots, x_{k}^{\alpha_{k}}\right)\right)\right|,
$$

Hence, applying Proposition 5, we get for $\alpha \in A_{k}$

$$
\begin{aligned}
\left\|I^{\alpha} f\right\|_{\Phi} & \leq(2 \pi)^{-k / 2}\|f\|_{\Phi}\left\|F_{k}^{-1}\left(h_{1}\left(x_{1}, \ldots, x_{k}\right) /\left(x_{1}^{\alpha_{1}} \ldots, x_{k}^{\alpha_{k}}\right)\right)\right\|_{L_{1}\left(\mathbb{R}^{k}\right)} \\
& =(2 \pi)^{-k / 2}\|f\|_{\Phi}\left\|F_{k}\left(h_{1}\left(x_{1}, \ldots, x_{k}\right) /\left(x_{1}^{\alpha_{1}} \ldots, x_{k}^{\alpha_{k}}\right)\right)\right\|_{L_{1}\left(\mathbb{R}^{k}\right)} .
\end{aligned}
$$

Since (33) and (34), the function $h_{1}\left(x_{1}, \ldots, x_{k}\right)$ satisfies the conditions in the Proposition 13. So,

$$
\varlimsup_{|\alpha| \rightarrow \infty}\left(\left(\inf _{\xi \in K_{\varepsilon}^{\prime}}\left|\xi^{\alpha}\right|\right)\left\|F_{k}\left(h_{1}\left(x_{1}, \ldots, x_{k}\right) /\left(x_{1}^{\alpha_{1}} \ldots, x_{k}^{\alpha_{k}}\right)\right)\right\|_{L_{1}\left(\mathbb{R}^{k}\right)}\right)^{1 /|\alpha|} \leq 1 .
$$

From this and (35) we get

$$
\left(A_{k}\right) \varlimsup_{|\alpha| \rightarrow \infty}\left(\left(\inf _{\xi \in K_{\varepsilon}}\left|\xi^{\alpha}\right|\right)\left\|I^{\alpha} f\right\|_{\Phi}\right)^{1 /|\alpha|} \leq 1 .
$$

By this and (31), we obtain

$$
\left(A_{k}\right) \varlimsup_{|\alpha| \rightarrow \infty}\left(\left(\inf _{\xi \in K}\left|\xi^{\alpha}\right|\right)\left\|I^{\alpha} f\right\|_{\Phi}\right)^{1 /|\alpha|} \leq \frac{\Delta}{\Delta-\varepsilon} .
$$

Letting $\varepsilon \rightarrow 0$, we have

$$
\left(A_{k}\right) \varlimsup_{|\alpha| \rightarrow \infty}\left(\left(\inf _{\xi \in \operatorname{supp} \hat{f}}\left|\xi^{\alpha}\right|\right)\left\|I^{\alpha} f\right\|_{\Phi}\right)^{1 /|\alpha|} \leq 1 .
$$

Step 3. We prove that

$$
\left(B_{k, u}\right) \varlimsup_{|\alpha| \rightarrow \infty}\left(\left(\inf _{\xi \in \operatorname{supp} \hat{f}}\left|\xi^{\alpha}\right|\right)\left\|I^{\alpha} f\right\|_{\Phi}\right)^{1 /|\alpha|} \leq 1,
$$

where $u \in \mathbb{Z}_{+}^{n-k}, B_{k, u}:=\left\{\alpha=\left(\alpha_{1}, \ldots, \alpha_{k}, u\right) \in \mathbb{Z}_{+}^{n}: \alpha_{1} \geq 3, \ldots, \alpha_{k} \geq 3\right\}$.

Put $a_{\alpha}=(0,0, \ldots, 0, u) \in \mathbb{Z}_{+}^{n}, b_{\alpha}=\left(\alpha_{1}, \alpha_{2}, \ldots, \alpha_{k}, 0, \ldots, 0\right) \in \mathbb{Z}_{+}^{n}, g=I^{a_{\alpha}} f$. Then $\alpha=a_{\alpha}+b_{\alpha}, \operatorname{supp} \hat{g}=\operatorname{supp} \hat{f}$, and from (22) we have $I^{\alpha} f=I^{b_{\alpha}} g$. By using Step 2 we have

$$
\left(A_{k}\right) \varlimsup_{|\alpha| \rightarrow \infty}\left(\left(\inf _{\xi \in \operatorname{supp} \hat{g}}\left|\xi^{b_{\alpha}}\right|\right)\left\|I^{b_{\alpha}} g\right\|_{\Phi}\right)^{1 /\left|b_{\alpha}\right|} \leq 1 .
$$

Hence,

$$
\left(B_{k, u}\right) \varlimsup_{|\alpha| \rightarrow \infty}\left(\left(\inf _{\xi \in \operatorname{supp} \hat{f}}\left|\xi^{b_{\alpha}}\right|\right)\left\|I^{\alpha} f\right\|_{\Phi}\right)^{1 /|\alpha|} \leq 1 .
$$

From this and the same argument to the proof of Proposition 13 when we derived (11) from 
(15) and (16), we obtain that

$$
\left(B_{k, u}\right) \varlimsup_{|\alpha| \rightarrow \infty}\left(\left(\inf _{\xi \in \operatorname{supp} \hat{f}}\left|\xi^{\alpha}\right|\right)\left\|I^{\alpha} f\right\|_{\Phi}\right)^{1 /|\alpha|} \leq 1
$$

and then Step 3 has been proved.

Let $0 \leq k \leq n, u \in \mathbb{Z}_{+}^{n-k}$ and $\left(i_{1}, \ldots, i_{n}\right)$ be a permutation of $(1,2, \ldots, n)$. We define $B_{k, u,\left(i_{1}, \ldots, i_{n}\right)}=\left\{\alpha=\left(\alpha_{1}, \ldots, \alpha_{n}\right) \in \mathbb{Z}_{+}^{n}: \alpha_{i_{1}} \geq 3, \ldots, \alpha_{i_{k}} \geq 3, \alpha_{i_{k+1}}=u_{1}, \ldots, \alpha_{i_{n}}=\right.$ $\left.u_{n-k}\right\}$. Then arguing similarly as in the proof of Step 3, we also have

$$
\left(B_{k, u,\left(i_{1}, \ldots, i_{n}\right)}\right) \varlimsup_{|\alpha| \rightarrow \infty}\left(\left(\inf _{\xi \in \operatorname{supp} \hat{f}}\left|\xi^{\alpha}\right|\right)\left\|I^{\alpha} f\right\|_{\Phi}\right)^{1 /|\alpha|} \leq 1 .
$$

We notice that $\mathbb{Z}_{+}^{n}$ is the finite union of $B_{k, u,\left(i_{1}, \ldots, i_{n}\right)}$, where $0 \leq k \leq n, u \in \mathbb{Z}_{+}^{n-k}, u \leq$ $(3,3, \ldots, 3),\left(i_{1}, \ldots, i_{n}\right)$ is a permutation of $(1,2, \ldots, n)$. Therefore,

$$
\varlimsup_{|\alpha| \rightarrow \infty}\left(\left(\inf _{\xi \in \operatorname{supp} \hat{f}}\left|\xi^{\alpha}\right|\right)\left\|I^{\alpha} f\right\|_{\Phi}\right)^{1 /|\alpha|} \leq 1 .
$$

The proof of (25) is complete.

Finally, we have to prove that

$$
\lim _{|\alpha| \rightarrow \infty}\left(\left(\inf _{\xi \in \operatorname{supp} \hat{f}}\left|\xi^{\alpha}\right|\right)\left\|I^{\alpha} f\right\|_{\Phi}\right)^{1 /|\alpha|} \geq 1 .
$$

Indeed, let $\sigma \in \operatorname{supp} \hat{f}$ and $\varepsilon>0$. Then there exists a function $\varphi \in C_{0}^{\infty}(B(\sigma, \varepsilon))$ such that $\langle\hat{f}, \varphi\rangle \neq 0$. Hence, it follows from Lemma 1 that

$$
0 \neq|\langle f, \hat{\varphi}\rangle|=\left|\left\langle D^{\alpha}\left(I^{\alpha} f\right), \hat{\varphi}\right\rangle\right|=\left|\left\langle I^{\alpha} f, D^{\alpha} \hat{\varphi}\right\rangle\right| \leq\left\|I^{\alpha} f\right\|_{\Phi}\left\|D^{\alpha} \hat{\varphi}\right\|_{\bar{\Phi}} .
$$

On the other hand, it follows from Theorem B that

$$
\lim _{|\alpha| \rightarrow \infty}\left(\frac{\left\|D^{\alpha} \hat{\varphi}\right\|_{\bar{\Phi}}}{\sup _{\xi \in \operatorname{supp} \varphi}\left|\xi^{\alpha}\right|}\right)^{1 /|\alpha|} \leq 1
$$

Therefore,

$$
\varlimsup_{|\alpha| \rightarrow \infty}\left(\frac{\left\|D^{\alpha} \hat{\varphi}\right\|_{\bar{\Phi}}}{\sup _{\xi \in B(\sigma, \varepsilon)}\left|\xi^{\alpha}\right|}\right)^{1 /|\alpha|} \leq 1 .
$$

From this and (37) we obtain

$$
\varliminf_{|\alpha| \rightarrow \infty}\left(\left(\sup _{\xi \in B(\sigma, \varepsilon)}\left|\xi^{\alpha}\right|\right)\left\|I^{\alpha} f\right\|_{\Phi}\right)^{1 /|\alpha|} \geq 1 .
$$


If $\xi \in B(\sigma, \varepsilon)$ then $\Delta<\left|\sigma_{j}\right|$ and $\left|\xi_{j}\right| \leq\left|\sigma_{j}\right|+\varepsilon$ for $j=1,2, \ldots, n$. Hence,

$$
\begin{aligned}
& \left(\left|\xi^{\alpha}\right|\right)^{1 /|\alpha|}=\left(\left|\sigma^{\alpha}\right|\right)^{1 /|\alpha|} \cdot\left(\left|\frac{\xi_{1}}{\sigma_{1}}\right|^{\alpha_{1}} \ldots\left|\frac{\xi_{n}}{\sigma_{n}}\right|^{\alpha_{n}}\right)^{1 /|\alpha|} \\
& \leq\left(\left|\sigma^{\alpha}\right|\right)^{1 /|\alpha|}\left(\left|\frac{\Delta+\varepsilon}{\Delta}\right|^{\alpha_{1}} \ldots\left|\frac{\Delta+\varepsilon}{\Delta}\right|^{\alpha_{n}}\right)^{1 /|\alpha|} \leq\left(\left|\sigma^{\alpha}\right|\right)^{1 /|\alpha|} \frac{\Delta+\varepsilon}{\Delta} .
\end{aligned}
$$

This gives

$$
\left(\sup _{\xi \in B(\sigma, \varepsilon)}\left|\xi^{\alpha}\right|\right)^{1 /|\alpha|} \leq \frac{\Delta+\varepsilon}{\Delta}\left|\sigma^{\alpha}\right|^{1 /|\alpha|} .
$$

Hence,

$$
\varliminf_{|\alpha| \rightarrow \infty}\left(\left|\sigma^{\alpha}\right| \cdot\left\|I^{\alpha} f\right\|_{\Phi}\right)^{1 /|\alpha|} \geq \frac{\Delta}{\Delta+\varepsilon} .
$$

Letting $\varepsilon \rightarrow 0$, we get

$$
\varliminf_{|\alpha| \rightarrow \infty}\left(\left|\sigma^{\alpha}\right| \cdot\left\|I^{\alpha} f\right\|_{\Phi}\right)^{1 /|\alpha|} \geq 1
$$

Suppose (36) is false. Then we can find an unbounded set $I_{1} \subset \mathbb{Z}_{+}^{n}, \lambda<1$ and a vector $\beta \geq 0,|\beta|=1$ such that

$$
\begin{aligned}
& \text { (I }) \lim _{|\alpha| \rightarrow \infty}\left(\left(\inf _{\xi \in \operatorname{supp} \hat{f}}\left|\xi^{\alpha}\right|\right)\left\|I^{\alpha} f\right\|_{\Phi}\right)^{1 /|\alpha|} \leq \lambda, \\
& \text { (I }) \lim _{|\alpha| \rightarrow \infty} \frac{\alpha}{|\alpha|}=\beta .
\end{aligned}
$$

We choose a number $\eta$ such that $1<\eta<\sqrt{\frac{1}{\lambda}}$. Because of $\left(I_{1}\right) \lim _{|\alpha| \rightarrow \infty} \frac{\alpha}{|\alpha|}=\beta$, we can deduce that there exists an unbounded subsequence $I_{2} \subset I_{1}$ such that

$$
\left(I_{2}\right)\left(\inf _{\xi \in \operatorname{supp} \hat{f}}\left|\xi^{\alpha}\right|\right)^{1 /|\alpha|} \geq \frac{1}{\eta} \inf _{\xi \in \operatorname{supp} \hat{f}}\left|\xi^{\beta}\right| .
$$

Indeed, it follows from the proof of (16) that

$$
\text { (I) } \lim _{|\alpha| \rightarrow \infty}\left(\inf _{\xi \in \operatorname{supp} \hat{f}}\left|\xi^{\alpha}\right|\right)^{1 /|\alpha|}=\inf _{\xi \in \operatorname{supp} \hat{f}}\left|\xi^{\beta}\right| .
$$

From this we have (40).

Combining (39)-(40), we get

$$
\text { (I2) } \lim _{|\alpha| \rightarrow \infty}\left(\inf _{\xi \in \operatorname{supp} \hat{f}}\left|\xi^{\beta}\right|\right)\left(\left\|I^{\alpha} f\right\|_{\Phi}\right)^{1 /|\alpha|} \leq \eta \lambda .
$$


Choose $\sigma \in \operatorname{supp} \hat{f}$ such that $\left|\sigma^{\beta}\right| \leq \eta \inf _{\xi \in \operatorname{supp} \hat{f}}\left|\xi^{\beta}\right|$. Then we have

$$
\text { (I2) } \varlimsup_{|\alpha| \rightarrow \infty}\left|\sigma^{\beta}\right|\left(\left\|I^{\alpha} f\right\|_{\Phi}\right)^{1 /|\alpha|} \leq \eta^{2} \lambda .
$$

From this and (38) we get

$$
\text { (I2) } \varlimsup_{|\alpha| \rightarrow \infty}\left|\sigma^{\beta-\frac{\alpha}{|\alpha|}}\right| \leq \eta^{2} \lambda<1 .
$$

This is a contradiction. So, (36) has been proved. Combing (25), (36), we obtain

$$
\lim _{|\alpha| \rightarrow \infty}\left(\left(\inf _{\xi \in \operatorname{supp} \hat{f}}\left|\xi^{\alpha}\right|\right)\left\|I^{\alpha} f\right\|_{\Phi}\right)^{1 /|\alpha|}=1 .
$$

The proof is complete.

Using Theorems 12 and $\mathrm{B}$, we have the following theorems:

THEOREM 14. Let $f \in L_{\Phi}\left(\mathbb{R}^{n}\right), f \not \equiv 0$, supp $\hat{f}$ be compact, $f$ has $(O)$-property and $\left(I^{\alpha} f\right)_{\alpha \in \mathbb{Z}_{+}^{n}} \subset L_{\Phi}\left(\mathbb{R}^{n}\right)$. Then

$$
\lim _{|\alpha| \rightarrow \infty}\left(\frac{\left\|D^{\alpha} f\right\|_{\Phi}}{\sup _{\xi \in \operatorname{supp} \hat{f}}\left|\xi^{\alpha}\right|}\right)^{1 /|\alpha|}=1
$$

and

$$
\lim _{|\alpha| \rightarrow \infty}\left(\left(\inf _{\xi \in \operatorname{supp} \hat{f}}\left|\xi^{\alpha}\right|\right)\left\|I^{\alpha} f\right\|_{\Phi}\right)^{1 /|\alpha|}=1
$$

THEOREM 15. Let $\left(I^{\alpha} f\right)_{\alpha \in \mathbb{Z}_{+}^{n}} \subset L_{\Phi}\left(\mathbb{R}^{n}\right)$ and $\sigma=\left(\sigma_{1}, \ldots, \sigma_{n}\right) \in \mathbb{R}_{+}^{n}$. Then $\operatorname{supp} \hat{f} \subset \prod_{k=1}^{n}\left(\left(-\infty,-\sigma_{k}\right] \cup\left[\sigma_{k},+\infty\right)\right)$ if and only if

$$
\varlimsup_{|\alpha| \rightarrow \infty}\left(\sigma^{\alpha}\left\|I^{\alpha} f\right\|_{\Phi}\right)^{1 /|\alpha|} \leq 1
$$

Proof. Necessary. It is clear from Theorem 12.

Sufficiency. Assume the contrary that there exists $\theta \in \operatorname{supp} \hat{f}, \theta \notin \prod_{k=1}^{n}\left(\left(-\infty,-\sigma_{k}\right] \cup\right.$ $\left.\left[\sigma_{k},+\infty\right)\right)$. Then there exists $j \in\{1,2, \ldots, n\}$ such that $\left|\theta_{j}\right|<\sigma_{j}$. Therefore,

$$
\begin{aligned}
1 & =\lim _{m \rightarrow \infty}\left(\left(\inf _{\xi \in \operatorname{supp} \hat{f}}\left|\xi^{m e_{j}}\right|\right)\left\|I^{m e_{j}} f\right\|_{\Phi}\right)^{1 / m} \leq \varlimsup_{m \rightarrow \infty}\left(\left|\theta_{j}^{m}\right| .\left\|I^{m e_{j}} f\right\|_{\Phi}\right)^{1 / m} \\
& =\frac{\left|\theta_{j}\right|}{\sigma_{j}} \varlimsup_{m \rightarrow \infty}\left(\sigma_{j}^{m}\left\|I^{m e_{j}} f\right\|_{\Phi}\right)^{1 / m} \leq \frac{\left|\theta_{j}\right|}{\sigma_{j}} .
\end{aligned}
$$


This is a contradiction. The proof is complete.

REMARK 16. Theorem 12 is not true if $f$ does not have $(O)$-property. It is clear because $\inf _{\xi \in \operatorname{supp} \hat{f}}\left|\xi^{\alpha}\right|=0$ for any $\alpha \in \mathbb{Z}_{+}^{n}$ such that $\alpha_{j} \geq 1, j=1, \ldots, n$.

We examine now behavior of $\left(\left\|I^{\alpha} f\right\|_{\Phi}\right)^{1 /|\alpha|}$ for functions $f$ not having $(O)$-property.

From the proof of Theorems 7, 11 and 12, we have the following result:

THEOREM 17. Let $f \in L_{\Phi}\left(\mathbb{R}^{n}\right), \operatorname{supp}_{k} \hat{f}:=\left\{\xi \in \mathbb{R}^{k}:(\xi, u) \in \operatorname{supp} \hat{f}\right.$ for some $u \in$ $\left.\mathbb{R}^{n-k}\right\}$ has (O)-property $\left(\right.$ in $\mathbb{R}^{k}$ ), and let $A=\left\{\alpha \in \mathbb{Z}_{+}^{n}: \quad \alpha_{k+1}=\cdots=\alpha_{n}=0\right\}$. Then there exists uniquely one sequence of primitives $\left(I^{\alpha} f\right)_{\alpha \in A} \subset L_{\Phi}\left(\mathbb{R}^{n}\right)$. Moreover, $\operatorname{supp} \widehat{I^{\alpha} f}=$ $\operatorname{supp} \hat{f} \quad \forall \alpha \in A$ and

$$
\text { (A) } \lim _{|\alpha| \rightarrow \infty}\left(\left(\inf _{\xi \in \operatorname{supp} \hat{f}}\left|\xi^{\alpha}\right|\right)\left\|I^{\alpha} f\right\|_{\Phi}\right)^{1 /|\alpha|}=1 .
$$

Further, we have:

PROPOSITION 18. Let $a>0, j \in\{1, \ldots, n\}$ and $A_{j}=\left\{\alpha \in \mathbb{Z}_{+}^{n}: \alpha_{j} \geq a|\alpha|\right\}$. Assume that there is an element in supp $\hat{f}$, the $j^{\text {th }}$ coordinate of which equals 0 . Then

$$
\left(A_{j}\right) \lim _{|\alpha| \rightarrow \infty}\left(\left\|I^{\alpha} f\right\|_{\Phi}\right)^{1 /|\alpha|}=\infty .
$$

Proof. We prove for $j=n$. We fix an element $\xi=\left(\xi_{1}, \ldots, \xi_{n-1}, 0\right) \in \operatorname{supp} \hat{f}$ and $\varepsilon \in(0,1)$ then there exists a function $\eta \in C_{0}^{\infty}(B(\xi, \varepsilon))$ such that $\langle\hat{f}, \eta\rangle \neq 0$. Hence,

$$
0 \neq|\langle\hat{f}, \eta\rangle|=|\langle f, \hat{\eta}\rangle|=\left|\left\langle D^{\alpha}\left(I^{\alpha} f\right), \hat{\eta}\right\rangle\right|=\left|\left\langle I^{\alpha} f, D^{\alpha} \hat{\eta}\right\rangle\right| \leq\left\|I^{\alpha} f\right\|_{\Phi}\left\|D^{\alpha} \hat{\eta}\right\|_{\bar{\Phi}} .
$$

Using Theorem B, we have

$$
\left(A_{n}\right) \varlimsup_{|\alpha| \rightarrow \infty}\left(\frac{\left\|D^{\alpha} \hat{\eta}\right\|_{\bar{\Phi}}}{\varepsilon^{\alpha_{n}} \prod_{j=1}^{n-1}\left(\left|\xi_{j}\right|+1\right)^{\alpha_{j}}}\right)^{1 /|\alpha|} \leq 1 .
$$

Therefore,

$$
\left(A_{n}\right) \varlimsup_{|\alpha| \rightarrow \infty} \frac{\left\|D^{\alpha} \hat{\eta}\right\|_{\bar{\Phi}}^{1 /|\alpha|}}{\varepsilon^{a} \prod_{j=1}^{n-1}\left(\left|\xi_{j}\right|+1\right)} \leq 1 .
$$

So,

$$
\left(A_{n}\right) \underset{|\alpha| \rightarrow \infty}{\lim _{|\alpha| \rightarrow}}\left(\left\|I^{\alpha} f\right\|_{\Phi}\right)^{1 /|\alpha|} \geq \varepsilon^{-a} \prod_{j=1}^{n-1}\left(\left|\xi_{j}\right|+1\right)^{-1}
$$


Letting $\varepsilon \rightarrow 0$, we get

$$
\left(A_{n}\right) \lim _{|\alpha| \rightarrow \infty}\left(\left\|I^{\alpha} f\right\|_{\Phi}\right)^{1 /|\alpha|}=\infty .
$$

The proof is complete.

THEOREM 19. Assume that for any $j \in\{1,2, \ldots, n\}$ there is an element in supp $\hat{f}$, the $j^{\text {th }}$ coordinate of which equals 0 . Then

$$
\lim _{|\alpha| \rightarrow \infty}\left(\left\|I^{\alpha} f\right\|_{\Phi}\right)^{1 /|\alpha|}=\infty
$$

Proof. For $1 \leq j \leq n$ we define

$$
A_{j}:=\left\{\alpha \in \mathbb{Z}_{+}^{n}: \quad \alpha_{j} \geq \frac{1}{n}|\alpha|\right\} .
$$

Then it follows from Proposition 18 that

$$
\left(A_{j}\right) \lim _{|\alpha| \rightarrow \infty}\left(\left\|I^{\alpha} f\right\|_{\Phi}\right)^{1 /|\alpha|}=\infty .
$$

From this and $\mathbb{Z}_{+}^{n}=\cup_{j=1}^{n} A_{j}$, we get

$$
\lim _{|\alpha| \rightarrow \infty}\left(\left\|I^{\alpha} f\right\|_{\Phi}\right)^{1 /|\alpha|}=\infty .
$$

The proof is complete.

We consider now the case when $f$ does not have $(O)$-property and not satisfies the condition in Theorem 19. The following theorem is clear from the proofs of Theorems 7, 11, 12 and Theorem 19:

THEOREM 20. Let $f \in L_{\Phi}\left(\mathbb{R}^{n}\right)$ and $1 \leq k<n$. Assume that for any $j \in\{k+$ $1, k+2, \ldots, n\}$ there is an element in $\operatorname{supp} \hat{f}$, the $j^{\text {th }}$ coordinate of which equals 0 , and $\left\{\xi \in \mathbb{R}^{k}:\right.$ there exists $u \in \mathbb{R}^{n-k}$ such that $\left.(\xi, u) \in \operatorname{supp} \hat{f}\right\} \subset\left(\mathbb{R}^{k}, \Delta\right)$ for some $\Delta>0$. Then

$$
\text { (A) } \lim _{|\alpha| \rightarrow \infty}\left(\left(\inf _{\xi \in \operatorname{supp} \hat{f}}\left|\xi^{\alpha}\right|\right)\left\|I^{\alpha} f\right\|_{\Phi}\right)^{1 /|\alpha|}=1 \text {, }
$$

where $A=\left\{\alpha \in \mathbb{Z}_{+}^{n}: \quad \alpha_{k+1}=\cdots=\alpha_{n}=0\right\}$ and

$$
\text { (B) } \lim _{|\alpha| \rightarrow \infty}\left(\left\|I^{\alpha} f\right\|_{\Phi}\right)^{1 /|\alpha|}=\infty
$$


here $B=\left\{\alpha \in \mathbb{Z}_{+}^{n}: \quad \alpha_{1}=\cdots=\alpha_{k}=0\right\}$.

Moreover, there doesn't exist the limit

$$
\lim _{|\alpha| \rightarrow \infty}\left(\left\|I^{\alpha} f\right\|_{\Phi}\right)^{1 /|\alpha|} .
$$

Proof. We have only to show that the limit doesn't exist. From the proof of Theorem 12 we have

$$
\text { (A) } \varlimsup_{|\alpha| \rightarrow \infty}\left(\left(\inf _{\xi \in \operatorname{supp} \hat{f}}\left|\xi_{1}^{\alpha_{1}} \ldots \xi_{k}^{\alpha_{k}}\right|\right)\left\|I^{\alpha} f\right\|_{\Phi}\right)^{1 /|\alpha|} \leq 1
$$

Therefore,

$$
\text { (A) } \varlimsup_{|\alpha| \rightarrow \infty}\left(\left\|I^{\alpha} f\right\|_{\Phi}\right)^{1 /|\alpha|} \leq \Delta^{-1}<\infty
$$

while

$$
\text { (B) } \lim _{|\alpha| \rightarrow \infty}\left(\left\|I^{\alpha} f\right\|_{\Phi}\right)^{1 /|\alpha|}=\infty
$$

The proof is complete.

Let $\Phi$ be an arbitrary Young function. We write $\Phi \in \Delta_{2}$ if there exists $C>0$ such that $\Phi(2 t) \leq C \Phi(t) \quad \forall t \in \mathbb{R}_{+}$. In conclusion, we give the following result:

THEOREM 21. Let $\Phi$ be an arbitrary Young function, $\Phi \in \Delta_{2}, \sigma=\left(\sigma_{1}, \ldots, \sigma_{n}\right) \in$ $\mathbb{R}_{+}^{n}, f \in L_{\Phi}\left(\mathbb{R}^{n}\right)$ and $\operatorname{supp} \hat{f} \subset \prod_{k=1}^{n}\left(\left(-\infty,-\sigma_{k}\right] \cup\left[\sigma_{k},+\infty\right)\right)$. Then

$$
\lim _{|\alpha| \rightarrow \infty} \sigma^{\alpha}\left\|I^{\alpha} f\right\|_{\Phi}=0
$$

To obtain Theorem 21 we recall the following result [11, 28]:

Bohr-Favard inequality. Let $\sigma>0, f \in C^{m}(\mathbb{R}), D^{m} f \in L_{\infty}(\mathbb{R})$ and supp $\hat{f} \subset$ $(-\infty,-\sigma] \cup[\sigma,+\infty)$. Then $f \in L_{\infty}(\mathbb{R})$ and

$$
\sigma^{m}\|f\|_{\infty} \leq K_{m}\left\|D^{m} f\right\|_{\infty},
$$

where the Favard constants $K_{m}$ are best possible and have the following properties

$$
1=K_{0} \leq K_{2}<\cdots<\frac{4}{\pi}<\cdots<K_{3} \leq K_{1}=\frac{\pi}{2} .
$$

By applying consecutively the Bohr-Favard inequality for $L_{\infty}(\mathbb{R})$ to each variable we have Bohr-Favard inequality for $L_{\infty}\left(\mathbb{R}^{n}\right)$, and then using the same arguments as in $[18,19]$ we get the following inequality for Orlicz spaces. 
Bohr-Favard inequality for Orlicz spaces. Let $f$ and its derivative $D^{\alpha} f$ be in $L_{\Phi}\left(\mathbb{R}^{n}\right)$ and $\operatorname{supp} \hat{f} \subset \prod_{k=1}^{n}\left(-\infty,-\sigma_{k}\right] \cup\left[\sigma_{k},+\infty\right), \sigma=\left(\sigma_{1}, \ldots, \sigma_{n}\right) \in \mathbb{R}_{+}^{n}$. Then

$$
\sigma^{\alpha}\|f\|_{\Phi} \leq K_{\alpha_{1}} \ldots K_{\alpha_{n}}\left\|D^{\alpha} f\right\|_{\Phi} \leq(\pi / 2)^{n}\left\|D^{\alpha} f\right\|_{\Phi} .
$$

Proof of Theorem 21. Because $\Phi \in \Delta_{2}$, for any $\varepsilon>0$, it is known that there exists a number $\lambda>1$ such that

$$
\|f(x)-f(\lambda x)\|_{\Phi} \leq \varepsilon .
$$

Put $h(x)=f(\lambda x)$. Then $\|f-h\|_{\Phi} \leq \varepsilon$ and

$$
\operatorname{supp} \hat{h}=\lambda \operatorname{supp} \hat{f} \subset \prod_{k=1}^{n}\left(\left(-\infty,-\lambda \sigma_{k}\right] \cup\left[\lambda \sigma_{k},+\infty\right)\right) .
$$

Applying Theorem 11, we obtain supp $\widehat{I^{\alpha} h}=\operatorname{supp} \widehat{h}$ and then

$$
\operatorname{supp} \widehat{I^{\alpha} h} \subset \prod_{k=1}^{n}\left(\left(-\infty,-\lambda \sigma_{k}\right] \cup\left[\lambda \sigma_{k},+\infty\right)\right) .
$$

Taking account of $D^{\alpha}\left(I^{\alpha} h\right)=h$ and applying the Bohr-Favard inequality for Orlicz space, we get

$$
(\lambda \sigma)^{\alpha}\left\|I^{\alpha} h\right\|_{\Phi} \leq(\pi / 2)^{n}\|h\|_{\Phi} .
$$

Since (42), supp $\widehat{f-h} \subset \prod_{k=1}^{n}\left(\left(-\infty,-\sigma_{k}\right] \cup\left[\sigma_{k},+\infty\right)\right)$. Then it follows from the Bohr-Favard for Orlicz space inequality that

$$
\sigma^{\alpha}\left\|I^{\alpha}(f-h)\right\|_{\Phi} \leq(\pi / 2)^{n}\|f-h\|_{\Phi} .
$$

Therefore,

$$
\begin{aligned}
\sigma^{\alpha}\left\|I^{\alpha} f\right\|_{\Phi} & \leq \sigma^{\alpha}\left\|I^{\alpha}(f-h)\right\|_{\Phi}+\sigma^{\alpha}\left\|I^{\alpha} h\right\|_{\Phi} \\
& \leq(\pi / 2)^{n}\|f-h\|_{\Phi}+(\pi / 2)^{n} \lambda^{-|\alpha|}\|h\|_{\Phi} \\
& \leq(\pi / 2)^{n} \varepsilon+(\pi / 2)^{n} \lambda^{-|\alpha|}\|h\|_{\Phi}
\end{aligned}
$$

for all $\alpha \in \mathbb{Z}_{+}^{n}$. Hence,

$$
\varlimsup_{|\alpha| \rightarrow \infty} \sigma^{\alpha}\left\|I^{\alpha} f\right\|_{\Phi} \leq(\pi / 2)^{n} \varepsilon .
$$

Letting $\varepsilon \rightarrow 0$, we get (41). The proof is complete.

Acknowledgement. This research is funded by Vietnam National Foundation for Science and Technology Development (NAFOSTED) under grant number 101.01-2011.32. 
A part of this work was done when the authors were working at the Vietnam Institute for Advanced Study in Mathematics (VIASM). The authors would like to thank the VIASM for providing a fruitful research environment and working condition. The authors would like to thank the referees for careful reading of our manuscript and the valuable remarks and suggestions.

\section{References}

[ 1 ] L.D. Abreu, Real Paley-Wiener theorems for the Koornwinder-Swarttouw $q$-Hankel transform, J. Math. Anal. Appl. 334 (2007), 223-231.

[ 2 ] E. Albrecht and Werner J. Ricker, Functional calculi and decomposability of unbounded multiplier operators in $L^{p}\left(\mathbb{R}^{N}\right)$, Proc. Edinburgh Math. Soc. 38 (1995), 151-166.

[3] E. Albrecht and W. J. RicKeR, Local spectral properties of certain matrix differential operators in $L^{p}\left(\mathbb{R}^{N}\right)^{m}$, J. Operator Theory 35 (1996), 3-37.

[ 4 ] N.B. Andersen, On real Paley-Wiener theorems for certain integral transforms, J. Math. Anal. Appl. 288 (2003), 124-135.

[ 5 ] N.B. Andersen, A simple proof of a Paley-Wiener type theorem for the Chébli-Trimèche transform, Publ. Math. Debrecen 64 (2004), 473-479.

[6] N.B. Andersen, Real Paley-Wiener theorems for the inverse Fourier transform on a Riemannian symmetric space, Pacific J. Math. 213 (2004), 1-13.

[ 7 ] N.B. Andersen, Real Paley-Wiener theorems, Bull. London Math. Soc. 36 (2004), 504-508.

[ 8 ] N.B. Andersen and M. DE Jeu, Elementary proofs of Paley-Wiener theorems for the Dunkl transform on the real line, Int. Math. Res. Notices 30 (2005), 1817-1831.

[9] N.B. Andersen, Real Paley-Wiener theorems for the Hankel transform, J. Fourier Anal. Appl. 12 (2006), $17-25$.

[10] N.B. Andersen and M. DE Jeu, Real Paley-Wiener theorems and local spectral radius formulas, Trans. Amer. Math. Soc. 362 (2010), 3613-3640.

[11] H. Bohr, Ein allgemeiner Satz fiber die Integration eines trigonometrischen Polynoms, Prace Matem.-Fiz 43 (1935), 273-288.

[12] H.H. Bang, A property of infinitely differentiable functions, Proc. Amer. Math. Soc. 108 (1990), 73-76.

[13] H.H. BANG, Functions with bounded spectrum, Trans. Amer. Math. Soc. 347 (1995), 1067-1080.

[14] H.H. BANG, A property of entire functions of exponential type, Analysis 15 (1995), 17-23.

[15] H.H. BANG, The existence of a point spectral radius of pseudodifferential operators, Doklady Mathematics 53 (1996), 420-422.

[16] H.H. BANG, The study of the properties of functions belonging to an Orlicz space depending on the geometry of their spectra, Izv. Akad. Nauk Ser. Mat. 61 (1997), 163-198.

[17] H.H. BANG, A study of the properties of functions depending on the geometry of their spectrum, Doklady Akad. Nauk 355 (1997), 740-743.

[18] H.H. BANG, An inequality of Bohr and Favard for Orlicz spaces, Bull. Polish Acad. Sci. Math. 49 (2001), 381-387.

[19] H.H. BANG and M.T. ThU, A Gagliardo-Nirenberg inequality for Orlicz spaces, East J. Approx. 10 (2004), 371-377.

[20] H.H. BANG and V.N. HUY, Behavior of the sequence of norms of primitives of a function, J. Approximation Theory 162 (2010), 1178-1186.

[21] H.H. BANG and V.N. HUY, Behavior of the sequences of norms of primitives of functions depending on their spectrum, Doklady Akad. Nauk 440 (2011), 456-458.

[22] J.J. Betancor, J.D. Betancor and J.M.R. MÉndez, Paley-Wiener type theorems for Chébli- Trimèche 
transforms, Publ. Math. Debrecen 60 (2002), 347-358.

[23] S.T. ChEn, Geometry of Orlicz Spaces, Dissertationes Math. 356 (1996), 1-204.

[24] C. Chettaoui and K. Trimèche, New type Paley-Wiener theorems for the Dunkl transform on $\mathbb{R}$, Integral Transforms Spec. Funct. 14 (2003), 97-115.

[25] H. Garth Dales, Pietro Aeina, Jrg Eschmeier, Kjeld Laursen and George A. Willis, Introduction to Banach Algebras, Operators and Harmonic Analysis (2003), Cambridge University Press.

[26] M. DE JEU, Some remarks on a proof of geometrical Paley-Wiener theorems for the Dunkl transform, Integral Transforms and Special Functions 18 (2007), 383-385.

[27] M. DE JEU, Paley-Wiener theorems for the Dunkl transform, Trans. Amer. Math. Soc. 358 (2006), 4225-4250.

[28] L. Hörmander, A new generalization of an inequality of Bohr, Math. Scand. 2 (1954), 33-45.

[29] M.A. KrasnoselskiI and Y. B. RutickiI, Convex Functions and Orlicz Spaces, GITTL, Moscow, 1958, English Transl. Noordhoff, 1961.

[30] W. LuXemburg, Banach Function Spaces (Thesis), Technische Hogeschool te Delft., The Netherlands, 1955.

[31] L. Maligranda, Orlicz Spaces and Interpolation, Seminars in Math. 5, Univ. Estadual de Campinas, Campinas, SP, Brasil, 1989.

[32] J. MusielaK, Orlicz Spaces and Modular Spaces, Lecture Notes in Math. 1034, Springer-Verlag, Berlin, 1983.

[33] R. O’ NeIL, Fractional integration in Orlicz space I, Trans. Amer. Math. Soc. 115 (1965), 300-328.

[34] M.M. RaO and Z.D. Ren, Theory of Orlicz Spaces, Marcel Dekker, Inc., New York, 1991.

[35] M.M. RAO and Z.D. ReN, Applications of Orlicz Spaces, Marcel Dekker Inc., New York, 2002.

[36] V.K. TuAN, On the supports of functions, Numer. Funct. Anal. Optim. 20 (1999), 387-394.

[37] V.K. Tuan, Paley-Wiener-type theorems, Fract. Calc. Appl. Anal. 2 (1999), 135-144.

[38] V.K. TUAN and A. ZAYED, Paley-Wiener-type theorems for a class of integral transforms, J. Math. Anal. Appl. 266 (2002), 200-226.

[39] V.K. TUAN, Spectrum of signals, J. Fourier Anal. Appl. 7 (2001), 319-323.

[40] V.S. Vladimirov, Methods of the theory of Generalized Functions, Taylor \& Francis, London, New York, 2002.

Present Addresses:

HA HUY BANG

INSTITUTE OF MATHEMATICS,

ViETNAMESE ACADEMY OF SCIENCE AND TECHNOLOGY,

18 Hoang Quoc Viet Street, Cau Giay, Hanoi, Vietnam.

e-mail: hhbang@math.ac.vn

VU NHAT HUY

DEPARTMENT OF MATHEMATICS,

COLlege of SCIENCE,

VIETNAM NATIONAL UNIVERSITY,

334 Nguyen Trai Street, Thanh Xuan, Hanoi, Vietnam.

e-mail:nhat_huy85@yahoo.com 